\title{
Full-semiparametric-likelihood-based inference for non-ignorable missing data
}

\author{
Yukun Liu, Pengfei Li, and Jing Qin*
}

\begin{abstract}
During the past few decades, missing-data problems have been studied extensively, with a focus on the ignorable missing case, where the missing probability depends only on observable quantities. By contrast, research into non-ignorable missing data problems is quite limited. The main difficulty in solving such problems is that the missing probability and the regression likelihood function are tangled together in the likelihood presentation, and the model parameters may not be identifiable even under strong parametric model assumptions. In this paper we discuss a semiparametric model for non-ignorable missing data and propose a maximum full semiparametric likelihood estimation method, which is an efficient combination of the parametric conditional likelihood and the marginal nonparametric biased sampling likelihood. The extra marginal likelihood contribution can not only produce efficiency gain but also identify the underlying model parameters without additional assumptions. We further show that the proposed estimators for the underlying parameters and the response mean are semiparametrically efficient. Extensive simulations and a real data analysis demonstrate the advantage of the proposed method over competing methods.
\end{abstract}

Key words and phrases: Density ratio model; Empirical likelihood; Identifiability; Maximum likelihood estimation; Non-ignorable missing data.

\footnotetext{
*Yukun Liu is Professor, School of Statistics, East China Normal University, 500 Dongchuan Road, Shanghai 200241, China (Email: ykliu@sfs.ecnu.edu.cn). Pengfei Li is Professor, Department of Statistics and Actuarial Sciences, University of Waterloo, Waterloo, ON, Canada, N2L 3G1 (Email: pengfei.li@uwaterloo.ca). Jing Qin is Mathematical Statistician, National Institute of Allergy and Infectious Diseases, National Institutes of Health, 6700B Rockledge Drive MSC 7609, Bethesda, MD 20892 (Email: jingqin@niaid.nih.gov).
} 


\section{Introduction}

Missing data is a ubiquitous problem in many areas, such as survey sampling, epidemiology, economics, sociology, and political science. Data may be missing because, for example, not every individual is sampled due to cost or inconvenience, or a sampled individual fails to report critical statistics. Missing-data problems have been studied extensively during the last few decades. Most research focuses on missing data that are ignorable or missing at random in the sense that the missing probability or propensity score is a function only of the observed data (Little and Rubin, 1987, 2002; Rubin, 1987).

Non-ignorable missing or missing-not-at-random data occur if the propensity score depends on the missing data, even conditionally on the observed data. Let $D$ be the missing indicator of the variable of interest $Y$ associated with some covariate variables $\mathbf{X}$, and $D=1$ if $Y$ is observed and $D=0$ otherwise. Non-ignorable missing implies that the propensity score $\operatorname{pr}(D=1 \mid \mathbf{x}, y)=\operatorname{pr}(D=1 \mid \mathbf{X}=\mathbf{x}, Y=y)$ depends on $y$ and possibly on $\mathbf{x}$. Inference for non-ignorable missing data is more challenging than that for ignorable missing data for at least two reasons. First, the equality $\operatorname{pr}(y \mid \mathbf{x}, D=1)=\operatorname{pr}(y \mid \mathbf{x}, D=0)$, which holds for ignorable missing data, does not hold for non-ignorable missing data. This implies that simply ignoring the missing data can lead to substantial selection bias (Groves et al., 2004). Second, unlike the ignorable missing case, the propensity score and the regression likelihood function are tangled together in non-ignorable missing-data problems, and hence cannot be estimated separately.

These challenges require new modelling strategies for non-ignorable missing data. The most popular strategy is to make assumptions about $\operatorname{pr}(D=1 \mid \mathbf{x}, y)$ and $\operatorname{pr}(y \mid \mathbf{x})$, based on the selection model factorization $\operatorname{pr}(y, D \mid \mathbf{x})=\operatorname{pr}(D \mid \mathbf{x}, y) \operatorname{pr}(y \mid \mathbf{x})$ of Little and Rubin (1987, 2002). Postulating parametric models (Greenless et al., 1982; Baker and Laird, 1988; Liu and Zhou, 2010) on both $\operatorname{pr}(D=1 \mid \mathbf{x}, y)$ and $\operatorname{pr}(y \mid \mathbf{x})$ is at risk of model mis-specification (Little, 1985). Meanwhile completely nonparametric models for $\operatorname{pr}(D=1 \mid \mathbf{x}, y)$ and $\operatorname{pr}(y \mid \mathbf{x})$ may make some model parameters not identifiable (Robins and Ritov, 1997). Attention has been paid to the case where one of these probabilities is parametric or semiparametric and the other is left unspecified. Tang et al. (2003) made a parametric assumption for $\operatorname{pr}(y \mid \mathbf{x})$ and left $\operatorname{pr}(D=1 \mid \mathbf{x}, y)$ unspecified. Many researchers considered a parametric model for $\operatorname{pr}(D=$ $1 \mid \mathbf{x}, y)$ and a nonparametric model for $\operatorname{pr}(y \mid \mathbf{x})$. See Qin et al. (2002); Chang and Kott (2008); 
Kott and Chang (2010); Morikawa and Kim (2016); Morikawa et al. (2017) and Ai et al. (2018). Kim and Yu (2011) proposed linking $\operatorname{pr}(y \mid \mathbf{x}, D=1)$ and $\operatorname{pr}(y \mid \mathbf{x}, D=0)$ by a semiparametric exponential tilting model, but a validation sample is required to estimate the tilting parameter in the semiparametric model. An alternative approach is to make parametric model assumptions on the observed $Y$ given X (Lee and Marsh, 2000; Riddles et al., 2016). An obvious advantage of this model over a completely parametric model for $\operatorname{pr}(y \mid \mathbf{x})$ is that it is checkable with available data.

Another layer of complication in non-ignorable missing-data problems is that the underlying parameters for the propensity score and regression likelihood function may not be identifiable even if both of them are assumed to follow completely parametric models. Wang et al. (2014) found that the model parameters can be identifiable given an instrument variable, which is correlated with the response variable but is independent of the propensity score conditional on the response variable. This strategy was further used by Zhao and Shao (2015) to identify the model parameters in generalized linear models based on non-ignorable missing data. Miao et al. (2016b) systematically investigated the identification of non-ignorable missing data via an ancillary variable, which is equivalent to Wang et al. (2014)'s instrument variable. Their general finding is that under non-ignorable missing data, many commonly used models are identifiable, and thus lack of identification is not an issue in many situations.

There have been many estimation approaches for identifiable model parameters developed in recent years, including pseudo-likelihood approaches (Tang et al., 2003; Zhao and Shao, 2015), empirical likelihood method (Zhao et al., 2013; Tang et al., 2014), and the generalized method of moments with an instrument variable (Wang et al., 2014; Shao and Wang, 2016; Shao, 2018). See Tang and Ju (2018) for a review of the most recent advances in dealing with nonignorable missing data. Under parametric models for both the propensity score and the observed $Y$ given $\mathbf{X}$, Riddles et al. (2016) proposed an estimating equation method based on Louis (1982)'s mean score equation, although their estimator is generally not efficient. When assuming a parametric model only for $\operatorname{pr}(D=1 \mid \mathbf{x}, y)$, Morikawa et al. (2017) proposed an estimating equation method by plugging-in a nonparametric estimator of $\operatorname{pr}(y \mid \mathbf{x}, D=1)$, which suffers from the curse of dimensionality and requires a bandwidth selection. Morikawa and Kim (2016) derived the semiparametric efficiency lower bound under the same assumptions. However, their semiparametrically efficient estimator requires explicit 
non-parametric estimation, also suffering from the curse of dimensionality and requiring a bandwidth selection. To avoid this dilemma, Ai et al. (2018) proposed a new estimation method based on the generalized method of moments with a diverging number of estimating equations. As the number of estimating equation increases, their estimator attains the semiparametric efficiency lower bound of Morikawa and Kim (2016). However, the constrained generalized method of moments may have numerical convergence problems, especially when some of the estimating equations are highly correlated.

In this paper, we consider parametric models for both $\operatorname{pr}(y \mid \mathbf{x}, D=1)$ and $\operatorname{pr}(D=1 \mid \mathbf{x}, y)$. In particular, we assume that $\operatorname{pr}(D=1 \mid \mathbf{x}, y)$ follows a logistic regression model,

$$
\operatorname{pr}(D=0 \mid \mathbf{x}, y)=\frac{\exp \left(\alpha^{*}+\mathbf{x}^{\top} \beta+y \gamma\right)}{1+\exp \left(\alpha^{*}+\mathbf{x}^{\top} \beta+y \gamma\right)},
$$

which is commonly used in practice. Under these assumptions, we find that the two distribution pairs $\{\operatorname{pr}(y \mid \mathbf{x}, D=1), \operatorname{pr}(y \mid \mathbf{x}, D=0)\}$ and $\{\operatorname{pr}(\mathbf{x} \mid D=1), \operatorname{pr}(\mathbf{x} \mid D=0)\}$ satisfy two density ratio models (Anderson, 1979, DRMs), see Equations (2.5) and (2.6), which share some key unknown parameters. We give an easy-to-check condition to verify the identifiability of the model parameters. This condition is satisfied by many existing identification conditions such as the existence of an instrument or ancillary variable (Wang et al., 2014; Miao et al., 2016a). For parameter estimation, the completely observed covariate data can be used to estimate the key unknown parameters, which can be further used to estimate $\operatorname{pr}(y \mid \mathbf{x}, D=0)$, since $\operatorname{pr}(y \mid \mathbf{x}, D=1)$ can be estimated directly using the conditional maximum likelihood method. These, together with the empirical distribution of $D$, lead to estimation of the conditional density $\operatorname{pr}(y \mid \mathbf{x})$; consequently the characteristics of $Y$ can be consistently estimated.

Given the completely observed covariate data and the fact that $\{\operatorname{pr}(\mathbf{x} \mid D=1), \operatorname{pr}(\mathbf{x} \mid D=$ 0)\} follows a DRM, we use Owen (1988, 2001)'s empirical likelihood (EL) to estimate the underlying parameters. Since Owen's seminal paper, the EL has become remarkably popular because it has many nice properties corresponding to those of parametric likelihood methods, e.g., it is range-preserving, transformation-respecting, and Bartlett correctable and it obeys Wilks' theorem (Hall and La Scala, 1990; DiCiccio et al., 1991; Qin and Lawless, 1994). The DRM-based EL has been demonstrated to be very flexible and efficient, and it has attracted much attention in recent decades; see Qin and Zhang (1997), Chen and Liu (2013), Cai et al. (2007), and the references therein. 
We show that the maximum EL estimators of the underlying parameters are asymptotically normal, and the EL ratio for all the parameters follows an asymptotically central chisquare distribution. This makes it much more convenient to conduct hypothesis testing or construct confidence intervals for these parameters. We propose a maximum likelihood estimator (MLE) for the marginal mean of the response variable, and we establish its asymptotic normality. We further show that the proposed MLEs for all parameters attain the corresponding semiparametric efficiency lower bounds under parametric assumptions for the propensity score and the conditional density of $Y$ given $\mathbf{X}$ and $D=1$. Compared with the existing methods, the proposed maximum semiparametric full likelihood approach has at least the following advantages:

1. It is able to identify the underlying parameters whether an instrument variable exists or not if the conditions in Proposition 1 are satisfied. The methods of Shao and Wang (2016), Riddles et al. (2016), Morikawa et al. (2017), Morikawa and Kim (2016) and Ai et al. (2018) all require an instrument variable. Further, it is able to produce consistent estimators for all the model parameters, if they are identifiable. Extra information about the parameter $\gamma$ in (1.1) is not needed.

2. It applies to data of any dimension and is free of bandwidth selection. The methods of Kim and Yu (2011), Shao and Wang (2016), Morikawa and Kim (2016), and Morikawa et al. (2017) all suffer from the curse of dimensionality and bandwidth selection, and may not work well for multivariate covariates. Ai et al. (2018)'s method has an increasing calculation burden as the number of estimating equation increases.

3. Existing methods handling non-ignorable missing-data problems under semiparametric setups are mainly based on estimating equations and may not be the most efficient in general. Since full likelihood approaches are generally the most efficient, it can be expected that the proposed maximum semiparametric full likelihood approach would outperform the existing methods. Even though Morikawa and Kim (2016) calculated the semiparametric efficiency lower bound with the specification of propensity score only, their lower bound is not achievable unless the conditional density of $Y$ given $(\mathbf{X}, D=1)$ is fully specified. In this paper we show that with the knowledge of $\operatorname{pr}(y \mid \mathbf{x}, D=1)$, Morikawa and Kim (2016)'s method is no longer optimal anymore. 
Our new lower bound is lower than theirs.

4. Our method is also applicable to retrospectively collected data. For example, when the number of nonresponse individuals (with $D=0$ ) is large, we can randomly select some covariate $\mathbf{x}$ from them to save cost. Based on this data together with the fully observed data, our method still provides valid inference about the underlying population. However, the existing methods may produce biased estimators because they are designed for prospective data.

The rest of this paper is organized as follows. In Section 2, we introduce the proposed model, show its equivalence to two DRMs, and provide sufficient conditions for the identifiability of the model parameters. Section 3 presents the proposed semiparametric DRM-based EL method and the resulting MLEs for the underlying parameters and the mean of the response variable; Their asymptotic normalities and semiparametric efficiencies are also established. Section 4 reports extensive simulation results. A real-life set of data is analyzed for illustration in Section 5. Section 6 provides concluding remarks. All technical details are given in the Appendix.

\section{Model and its identifiability}

\subsection{Model set-up}

Suppose $\left\{\left(y_{i}, \mathbf{x}_{i}, d_{i}\right), i=1, \ldots, n\right\}$ are $n$ independent and identically distributed copies of $(Y, \mathbf{X}, D)$, where the covariates $\mathbf{x}_{i}$ are always observed, and $y_{i}$ is observed if and only if $d_{i}=1$. We assume that the missing probability satisfies the logistic regression model in (1.1), i.e.,

$$
\operatorname{pr}(D=0 \mid \mathbf{x}, y)=\frac{\exp \left(\alpha^{*}+\mathbf{x}^{\top} \beta+y \gamma\right)}{1+\exp \left(\alpha^{*}+\mathbf{x}^{\top} \beta+y \gamma\right)}
$$

The parameter $\gamma$ is called the tilting parameter (Kim and Yu, 2011). It quantifies the extent to which the model departs from ignorable missing, and $\gamma=0$ corresponds to the ignorable missing-data case. We are interested in estimating the underlying parameters $\left(\alpha^{*}, \beta, \gamma\right)$ and the marginal mean $\mu$ of $Y$. 
Based on the observed data, the full likelihood is

$$
\prod_{i=1}^{n}\left[\left\{\operatorname{pr}\left(D=1 \mid \mathbf{x}_{i}, y_{i}\right) \operatorname{pr}\left(y_{i}, \mathbf{x}_{i}\right)\right\}^{d_{i}}\left\{\int \operatorname{pr}\left(D=0 \mid \mathbf{x}_{i}, y\right) \operatorname{pr}\left(y, \mathbf{x}_{i}\right) d y\right\}^{1-d_{i}}\right] .
$$

Unlike the case of ignorable missing, here $\operatorname{pr}(D=1 \mid \mathbf{x}, y)$ and $\operatorname{pr}(y, \mathbf{x})$ can not be separated and hence can not be separately estimated. To make inference based on the full likelihood, one may postulate parametric assumptions on $\operatorname{pr}(D=1 \mid y, \mathbf{x})$ and $\operatorname{pr}(y \mid \mathbf{x})$, which are sensitive to model mis-specification (Little, 1985; Kenward and Molenberghs, 1988).

We crack this nut by an alternative method. The logistic regression model (1.1) is equivalent to the two-sample DRM (Qin and Zhang, 1997)

$$
\operatorname{pr}(\mathbf{x}, y \mid D=0)=\exp \left(\alpha+\mathbf{x}^{\top} \beta+y \gamma\right) \operatorname{pr}(\mathbf{x}, y \mid D=1)
$$

where $\alpha=\alpha^{*}+\log \{\eta /(1-\eta)\}$ and $\eta=\operatorname{pr}(D=1)$ is the probability of being observed. Clearly, $\eta$ can be consistently estimated by data and is therefore identifiable. Then the identifiability of $\alpha^{*}$ is equivalent to that of $\alpha$.

Integrating out $y$, we have

$$
\operatorname{pr}(\mathbf{x} \mid D=0)=\exp \left(\alpha+\mathbf{x}^{\top} \beta\right) \operatorname{pr}(\mathbf{x} \mid D=1) \int \exp (y \gamma) \operatorname{pr}(y \mid \mathbf{x}, D=1) d y
$$

Therefore, the conditional densities of $Y=y$ given $(\mathbf{X}=\mathbf{x}, D=0)$ and given $(\mathbf{X}=\mathbf{x}, D=1)$ satisfy

$$
\operatorname{pr}(y \mid \mathbf{x}, D=0)=\frac{\operatorname{pr}(\mathbf{x}, y \mid D=0)}{\operatorname{pr}(\mathbf{x} \mid D=0)}=\frac{\exp (y \gamma) \operatorname{pr}(y \mid \mathbf{x}, D=1)}{\int \exp (y \gamma) \operatorname{pr}(y \mid \mathbf{x}, D=1) d y} .
$$

Although $\operatorname{pr}(y \mid \mathbf{x}, D=1)$ is directly estimable based on the observed $\left(y_{i}, \mathbf{x}_{i}\right)$ 's with $d_{i}=1$, it is impossible to estimate $\operatorname{pr}(y \mid \mathbf{x}, D=0)$ since $\gamma$ is unknown in general. As a consequence, the conditional approach is not viable, as demonstrated by Kim and Yu (2011), who rely on external data to identify $\gamma$. In practical applications, however, external data are often unavailable, which makes the estimation of $\gamma$ impossible.

Fortunately, the marginal information on the $\left(\mathbf{x}_{i}, d_{i}\right)$ 's can help to identify $\gamma$, which solves the thorny identifiability problem in non-ignorable missing-data problems. Since $\left(y_{i}, \mathbf{x}_{i}\right)^{\prime}$ 's with $d_{i}=1$ are available, without loss of generality, we can postulate a parametric model $f(y \mid \mathbf{x}, \xi)$ for $\operatorname{pr}(y \mid \mathbf{x}, D=1)$ with an identifiable parameter $\xi$. The parameter $\xi$ can be 
consistently estimated from the directly observed data. This parametric model together with Equation (2.4) implies two DRMs:

$$
\begin{aligned}
\operatorname{pr}(y \mid \mathbf{x}, D=0) & =\exp \{\gamma y-c(\mathbf{x}, \gamma, \xi)\} f(y \mid \mathbf{x}, \xi) \\
\operatorname{pr}(\mathbf{x} \mid D=0) & =\exp \left\{\alpha+\mathbf{x}^{\top} \beta+c(\mathbf{x}, \gamma, \xi)\right\} \operatorname{pr}(\mathbf{x} \mid D=1)
\end{aligned}
$$

where

$$
c(\mathbf{x}, \gamma, \xi)=\ln \left\{\int \exp (y \gamma) f(y \mid \mathbf{x}, \xi) d y\right\} .
$$

Equations (2.5)-(2.7) are the foundation of our inference method. We note that the second DRM involves all the underlying parameters in the model and is dependent only on $\operatorname{pr}(\mathbf{x} \mid D=$ $0)$ and $\operatorname{pr}(\mathbf{x} \mid D=1)$. Since the $\left(\mathbf{x}_{i}, d_{i}\right)$ 's with $d_{i}=0$ or 1 are not subject to missingness, the parameters can be consistently estimated by their maximum DRM-based EL estimators (Qin and Zhang, 1997) provided they are identifiable.

\subsection{Model identifiability}

Miao et al. (2016a) pointed out that even under full parametric models for $\operatorname{pr}(D=1 \mid \mathbf{x}, y)$ and $\operatorname{pr}(y \mid \mathbf{x})$, the underlying model parameters may not be identifiable. This phenomenon also arises under Model (2.6) , where even $\operatorname{pr}(\mathbf{x} \mid D=1)$ is completely known, the model parameters in (2.6) may not be identifiable. We present a simple-to-check sufficient condition for the identifiability of the underlying parameters in (2.6). We have assumed that $\xi$ is identifiable. Hence, we focus here on the identifiability of the parameters $\alpha, \beta$, and $\gamma$. Given the data $\left\{\left(\mathbf{x}_{i}, d_{i}\right), i=1, \ldots, n\right\}$, the conditional density functions $\operatorname{pr}(\mathbf{x} \mid D=0)$ and $\operatorname{pr}(\mathbf{x} \mid D=1)$ are clearly identifiable and can be consistently estimated by, for example, the kernel method. The $\log$ ratio $\log \{\operatorname{pr}(\mathbf{x} \mid D=0) / \operatorname{pr}(\mathbf{x} \mid D=1)\}$ is also identifiable. Since

$$
\log \{\operatorname{pr}(\mathbf{x} \mid D=0) / \operatorname{pr}(\mathbf{x} \mid D=1)\}=\alpha+\mathbf{x}^{\top} \beta+c(\mathbf{x}, \gamma, \xi),
$$

the model identification is equivalent to the identification of the parameters $\alpha, \beta$, and $\gamma$ in $\alpha+\mathbf{x}^{\top} \beta+c(\mathbf{x}, \gamma, \xi)$.

Proposition 1. Let $S$ be the common support of $\operatorname{pr}(\mathbf{x} \mid D=0)$ and $\operatorname{pr}(\mathbf{x} \mid D=1)$, and $\Omega=\left\{h(\mathbf{x}): S \mapsto \mathbb{R} \mid \exists(\alpha, \beta, \gamma)\right.$ such that $\left.h(\mathbf{x})=\alpha+\mathbf{x}^{\top} \beta+c(\mathbf{x}, \gamma, \xi) \forall \mathbf{x} \in S\right\}$. If for any $h(\mathbf{x}) \in \Omega$, there exists a unique $(\alpha, \beta, \gamma)$ such that $h(\mathbf{x})=\alpha+\mathbf{x}^{\top} \beta+c(\mathbf{x}, \gamma, \xi)$, then $(\alpha, \beta, \gamma)$ is identifiable. 
Next we apply the above proposition to some special cases. We need the concept of an instrument variable, which can be helpful to identify $\gamma$. Suppose $\mathbf{x}$ can be written as $\mathbf{x}=\left(z, u^{\top}\right)^{\top}$. If

$$
\operatorname{pr}(D=0 \mid z, u, y)=\operatorname{pr}(D=0 \mid u, y)=\frac{\exp \left(\alpha^{*}+u^{\top} \beta+y \gamma\right)}{1+\exp \left(\alpha^{*}+u^{\top} \beta+y \gamma\right)}
$$

and $\operatorname{pr}(y \mid \mathbf{x})=\operatorname{pr}(y \mid z, u)$ depends on $z$ and possibly on $u$, then $z$ is an instrument variable. That is, an instrument variable is defined to be a covariate that does not affect the missingness but may affect the conditional distribution of the response variable.

With the above preparation and Proposition 1, we find that $(\alpha, \beta, \gamma)$ is identifiable in the following two cases.

Corollary 1. Suppose the logistic regression model in (1.1) holds and that the density function of $Y$ given $(\mathbf{X}=\mathbf{x}, D=1)$ is $f(y \mid \mathbf{x}, \xi)$. (a) If there exists an instrument variable $z$ in $\mathbf{x}$, then $(\alpha, \beta, \gamma)$ is identifiable. (b) Assume that the set $S$ in Proposition 1 contains an open set, and $c(\mathbf{x}, \gamma, \xi)$ can be expressed as $c(\mathbf{x}, \gamma, \xi)=\sum_{i=1}^{k} a_{i}(\gamma) g_{i}(\mathbf{x})+a_{k+1}(\gamma)+\mathbf{x}^{\top} a_{k+2}(\gamma)$ for some positive integer $k$, and continuous functions $a_{i}(\gamma)(i=1, \ldots, k+2)$ and $g_{i}(\mathbf{x})(i=1, \ldots, k)$, where $1, \mathbf{x}, g_{1}(\mathbf{x}), \ldots, g_{k}(\mathbf{x})$ are linearly independent, and $a_{j}(\gamma)(j=1, \ldots, k)$ are not equal to the zero functions. If $\left(a_{1}\left(\gamma_{1}\right), \ldots, a_{k}\left(\gamma_{1}\right)\right) \neq\left(a_{1}\left(\gamma_{2}\right), \ldots, a_{k}\left(\gamma_{2}\right)\right)$ for any $\gamma_{1} \neq \gamma_{2}$, then $(\alpha, \beta, \gamma)$ is identifiable.

As an application of the above results, we consider the normal model in which $f(y \mid \mathbf{x}, \xi)$ is the density function of $N\left(\mu(\mathbf{x}, \xi), \sigma^{2}(\mathbf{x}, \xi)\right)$. Direct calculations give $c(\mathbf{x}, \gamma, \xi)=\gamma \mu(\mathbf{x}, \xi)+$ $0.5 \gamma^{2} \sigma^{2}(\mathbf{x}, \xi)$. Further, assume $\mu(\mathbf{x}, \xi)=\mathbf{x}^{\top} b_{1}(\xi)+b_{2}(\xi) \mathbf{x}^{\top} \mathbf{x}$ and $\sigma^{2}(\mathbf{x}, \xi)=\exp \left\{b_{3}(\xi)+\right.$ $\left.\mathbf{x}^{\top} b_{4}(\xi)\right\}$ for nonzero functions $b_{i}(\xi)$. We have the following observations:

(I) If $b_{2}(\xi) \neq 0$, then according to Corollary $1,(\alpha, \beta, \gamma)$ is identifiable.

(II) If $b_{2}(\xi)=0$ and $b_{4}(\xi)=0$, then

$$
\alpha+\mathbf{x}^{\top} \beta+c(\mathbf{x}, \gamma, \xi)=\alpha+0.5 \gamma^{2} \exp \left\{b_{3}(\xi)\right\}+\mathbf{x}^{\top}\left\{\beta+\gamma b_{1}(\xi)\right\}
$$

which together with Lemma 1 implies that $(\alpha, \beta, \gamma)$ is not identifiable.

(III) If $b_{2}(\xi)=0$ and $b_{4}(\xi) \neq 0$, then

$$
\alpha+\mathbf{x}^{\top} \beta+c(\mathbf{x}, \gamma, \xi)=\alpha+\mathbf{x}^{\top}\left\{\beta+\gamma b_{1}(\xi)\right\}+0.5 \gamma^{2} \exp \left\{b_{3}(\xi)+\mathbf{x}^{\top} b_{4}(\xi)\right\}
$$


If further $\gamma=0$, then Proposition 1 implies that $(\alpha, \beta, \gamma)$ is identifiable. Otherwise, $(\alpha, \beta, \gamma)$ is not identifiable.

\section{Semiparametric empirical likelihood inference}

\subsection{Empirical likelihood}

Suppose there are $n_{1}$ completely observed data and $n_{2}$ partially observed data. Without loss of generality, we assume that $d_{i}=1, i=1, \ldots, n_{1}$ and $d_{i}=0, i=n_{1}+1, \ldots, n$. The full likelihood in (2.2) can be written as

$$
\prod_{i=1}^{n_{1}}\left\{\operatorname{pr}\left(y_{i} \mid \mathbf{x}_{i}, D=1\right) \operatorname{pr}\left(\mathbf{x}_{i} \mid D=1\right) \operatorname{pr}(D=1)\right\} \cdot \prod_{i=n_{1}+1}^{n}\left\{\operatorname{pr}\left(\mathbf{x}_{i} \mid D=0\right) \operatorname{pr}(D=0)\right\} .
$$

Let $\theta=\left(\alpha, \beta^{\top}, \gamma, \xi^{\top}\right)^{\top}$ and $t(\mathbf{x}, \theta)=\alpha+\mathbf{x}^{\top} \beta+c(\mathbf{x}, \gamma, \xi)$. Since $\operatorname{pr}(y \mid \mathbf{x}, D=1)=f(y \mid \mathbf{x}, \xi)$ by assumption, it follows from $\eta=\operatorname{pr}(D=1)$ and Equation (2.6) that the full log-likelihood is $\tilde{\ell}=\ell_{1}(\eta)+\tilde{\ell}_{2}$, where

$$
\ell_{1}(\eta)=n_{1} \log (\eta)+\left(n-n_{1}\right) \log (1-\eta)
$$

is the marginal likelihood based on the $d_{i}$ 's, and

$$
\tilde{\ell}_{2}=\sum_{i=1}^{n_{1}} \log \left\{f\left(y_{i} \mid \mathbf{x}_{i}, \xi\right)\right\}+\sum_{i=n_{1}+1}^{n} t\left(\mathbf{x}_{i}, \theta\right)+\sum_{i=1}^{n} \log \left\{\operatorname{pr}\left(\mathbf{x}_{i} \mid D=1\right)\right\}
$$

is a conditional likelihood given the $d_{i}$ 's.

We leave the conditional density $\operatorname{pr}(\mathbf{x} \mid D=1)$ completely unspecified, and use the celebrated EL method of Owen (1988, 1990) to handle it. Let $p_{i}=\operatorname{pr}\left(\mathbf{x}_{i} \mid D=1\right)=d F\left(\mathbf{x}_{i} \mid D=1\right)$, where $F(\mathbf{x} \mid D=1)$ is the cumulative distribution function corresponding to the density $\operatorname{pr}(\mathbf{x} \mid D=1)$. Following the principle of EL, $\tilde{\ell}_{2}$ becomes an empirical log-likelihood

$$
\tilde{\ell}_{2}=\sum_{i=1}^{n_{1}} \log \left\{f\left(y_{i} \mid \mathbf{x}_{i}, \xi\right)\right\}+\sum_{i=n_{1}+1}^{n} t\left(\mathbf{x}_{i}, \theta\right)+\sum_{i=1}^{n} \log \left(p_{i}\right)
$$

where the $p_{i}$ 's are subject to the constraints

$$
p_{i} \geq 0, \quad \sum_{i=1}^{n} p_{i}=1, \quad \sum_{i=1}^{n} p_{i}\left[\exp \left\{t\left(\mathbf{x}_{i}, \theta\right)\right\}-1\right]=0 .
$$


Maximizing $\tilde{\ell}_{2}$ with respect to the $p_{i}$ 's, we arrive at

$$
p_{i}=\frac{1}{n} \frac{1}{1+\lambda\left[\exp \left\{t\left(\mathbf{x}_{i}, \theta\right)\right\}-1\right]}
$$

where $\lambda$ is the solution to

$$
\sum_{i=1}^{n} \frac{\exp \left\{t\left(\mathbf{x}_{i}, \theta\right)\right\}-1}{1+\lambda\left[\exp \left\{t\left(\mathbf{x}_{i}, \theta\right)\right\}-1\right]}=0
$$

Substituting these $p_{i}$ 's into $\tilde{\ell}_{2}$ leads to the profile log-likelihood of $\theta$,

$$
\ell_{2}(\theta)=\sum_{i=1}^{n_{1}} \log \left\{f\left(y_{i} \mid \mathbf{x}_{i}, \xi\right)\right\}+\sum_{i=n_{1}+1}^{n} t\left(\mathbf{x}_{i}, \theta\right)-\sum_{i=1}^{n} \log \left\{1+\lambda\left[\exp \left\{t\left(\mathbf{x}_{i}, \theta\right)\right\}-1\right]\right\} .
$$

The profile log-likelihood of $(\eta, \theta)$ is then defined as

$$
\ell(\eta, \theta)=\ell_{1}(\eta)+\ell_{2}(\theta) .
$$

\subsection{Estimation of the underlying parameters}

With the profile log-likelihood of $(\eta, \theta)$ in (3.10), the MLE of $(\eta, \theta)$ is

$$
(\hat{\eta}, \hat{\theta})=\arg \max _{\eta, \theta} \ell(\eta, \theta)
$$

Equivalently, $\hat{\eta}$ maximizes $\ell_{1}(\eta)$, which gives $\hat{\eta}=n_{1} / n$, and $\hat{\theta}=\left(\hat{\alpha}, \hat{\beta}^{\top}, \hat{\gamma}, \hat{\xi}^{\top}\right)^{\top}=\arg \max _{\theta} \ell_{2}(\theta)$. The likelihood ratio function of $\theta$ is defined as

$$
R(\theta)=2\left\{\max _{\eta, \theta} \ell(\eta, \theta)-\max _{\eta} \ell(\eta, \theta)\right\}=2\left\{\ell_{2}(\hat{\theta})-\ell_{2}(\theta)\right\} .
$$

Next we study the large-sample properties of the MLE and the likelihood ratio. Denote the truth of $(\eta, \theta)$ by $\left(\theta_{0}, \eta_{0}\right)$ with $\theta_{0}=\left(\alpha_{0}, \beta_{0}^{\top}, \gamma_{0}, \xi_{0}^{\top}\right)^{\top}$ and $\eta_{0} \in(0,1)$. Define

$$
\pi(\mathbf{x} ; \theta, \eta)=\frac{(1-\eta) \exp \{t(\mathbf{x}, \theta)\}}{\eta+(1-\eta) \exp \{t(\mathbf{x}, \theta)\}}
$$

and we write $\pi(\mathbf{x})=\pi\left(\mathbf{x} ; \theta_{0}, \eta_{0}\right)$ for abbreviation. Let $d_{\theta}$ denote the dimension of $\theta$ and $\mathbf{e}_{1}$ be a $d_{\theta} \times 1$ vector with the first component being 1 and the remaining components 0 . Finally, define

$$
V=\mathbb{E}\left[\{1-\pi(\mathbf{X})\} \pi(\mathbf{X})\left\{\nabla_{\theta} t\left(\mathbf{X}, \theta_{0}\right)\right\}^{\otimes 2}\right]+\mathbb{E}\left[D I_{e}\left\{\nabla_{\xi} f\left(Y \mid \mathbf{X}, \xi_{0}\right)\right\}^{\otimes 2} I_{e}^{\top}\right],
$$

where $\nabla_{\theta}$ is the differentiation operator with respect to $\theta, I_{e}^{\top}=\left(0_{d_{\xi} \times\left(2+d_{\beta}\right)}, I_{d_{\xi} \times d_{\xi}}\right)$, and $B^{\otimes 2}=B B^{\top}$ for any matrix or vector $B$. 
Theorem 1. Assume Conditions A1-A4 in Appendix 1. Suppose that the logistic regression model in (1.1) holds with $\left(\alpha_{0}, \beta_{0}, \gamma_{0}\right)$ in place of $(\alpha, \beta, \gamma)$, and that the density function of $Y$ given $(\mathbf{X}=\mathbf{x}, D=1)$ is $f\left(y \mid \mathbf{x}, \xi_{0}\right)$. Further, assume that $\theta$ is identifiable. Then as $n \rightarrow \infty$, (1) $\sqrt{n}\left(\hat{\theta}-\theta_{0}\right) \rightarrow N\left(0, V^{-1}-\left\{\eta_{0}\left(1-\eta_{0}\right)\right\}^{-1} \mathbf{e}_{1} \mathbf{e}_{1}^{\top}\right)$ in distribution with $V$ defined in (3.11); (2) $R\left(\theta_{0}\right) \rightarrow \chi_{d_{\theta}}^{2}$ in distribution.

Theorem 1 implies that the MLEs of all the parameters are asymptotically normal. The likelihood ratio for the parameters follows a central chisquare limiting distribution, which makes the resulting hypothesis testing or interval estimation about $\theta$ very convenient. Although the proposed approach is developed based on prospective data, we emphasize that it can also apply to retrospectively collected data. This is because the subsequent inferences are mainly based on $\ell_{2}$ or equivalently

$$
\tilde{\ell}_{2}=\log \left[\prod_{i=1}^{n_{1}}\left\{\operatorname{pr}\left(y_{i}, \mathbf{x}_{i} \mid D=1\right)\right\} \prod_{i=n_{1}+1}^{n}\left\{\operatorname{pr}\left(\mathbf{x}_{i} \mid D=0\right)\right],\right.
$$

which is actually a retrospective log-likelihood. If $\eta=\operatorname{pr}(D=1)$ or $\hat{\eta}$ is available, based on retrospectively collected data, the proposed approach can still make valid inference.

Given the MLE of all the underlying parameters, we are able to construct the MLE of the population mean $\mu$ of the response $Y$. Under our model, $\mu$ depends not only on the underlying parameters $\theta$ but also on $\operatorname{pr}(\mathbf{x} \mid D=1)$ or the corresponding cumulative distribution function $F(\mathbf{x} \mid D=1)$. With the MLEs $\hat{\theta}$ and $\hat{\eta}=n_{1} / n$, we show in the supplementary material that $\hat{\lambda}=n_{2} / n$, where $\hat{\lambda}$ satisfies (3.9) with $\hat{\theta}$ in the place of $\theta$. With (3.8), the MLE of $p_{i}$ is

$$
\hat{p}_{i}=\frac{1}{n} \frac{1}{1+\left(n_{2} / n\right)\left[\exp \left\{t\left(\mathbf{x}_{i}, \hat{\theta}\right)\right\}-1\right]}=\frac{1}{n} \frac{1}{\hat{\eta}+(1-\hat{\eta}) \exp \left\{t\left(\mathbf{x}_{i}, \hat{\theta}\right)\right\}} .
$$

Accordingly the MLE of $F(\mathbf{x} \mid D=1)$ is $\hat{F}(\mathbf{x} \mid D=1)=\sum_{i=1}^{n_{1}} \hat{p}_{i} I\left(\mathbf{x}_{i} \leq \mathbf{x}\right)$, where for two vectors $\mathbf{x}_{1}$ and $\mathbf{x}_{2}, \mathbf{x}_{1} \leq \mathbf{x}_{2}$ implies that the inequality holds elementwise. 


\subsection{Estimation of the response mean}

To obtain the MLE of the response mean $\mu$, we write $\mu$ in terms of the underlying parameters $\eta, \theta$, and $F(\mathbf{x} \mid D=1)$ as follows:

$$
\begin{aligned}
\mu= & \int_{y} \int_{\mathbf{X}} y \operatorname{pr}(y \mid \mathbf{x}, D=1) \operatorname{pr}(\mathbf{x} \mid D=1) \operatorname{pr}(D=1) d \mathbf{x} d y \\
& +\int_{y} \int_{\mathbf{X}} y \operatorname{pr}(y \mid \mathbf{x}, D=0) \operatorname{pr}(\mathbf{x} \mid D=0) \operatorname{pr}(D=0) d \mathbf{x} d y \\
= & \int_{y} \int_{\mathbf{X}} y \operatorname{pr}(y \mid \mathbf{x}, D=1) \operatorname{pr}(\mathbf{x} \mid D=1) \eta d \mathbf{x} d y \\
& +\int_{y} \int_{\mathbf{X}} y \exp \left(\alpha+\mathbf{x}^{\top} \beta+\gamma y\right) \operatorname{pr}(y \mid \mathbf{x}, D=1) \operatorname{pr}(\mathbf{x} \mid D=1)(1-\eta) d \mathbf{x} d y \\
= & \int_{\mathbf{X}}\left[\int_{y} y\left\{\eta+(1-\eta) \exp \left(\alpha+\mathbf{x}^{\top} \beta+\gamma y\right)\right\} f(y \mid \mathbf{x}, \xi) d y\right] d F(\mathbf{x} \mid D=1),
\end{aligned}
$$

where in the last step we replace $\operatorname{pr}(y \mid \mathbf{x}, D=1)$ and $\operatorname{pr}(\mathbf{x} \mid D=1) d \mathbf{x}$ by $f(y \mid \mathbf{x}, \xi)$ and $d F(\mathbf{x} \mid D=1)$, respectively. Then the MLE of $\mu$ is

$$
\begin{aligned}
\hat{\mu} & =\sum_{i=1}^{n} \hat{p}_{i}\left[\int_{y} y\left\{\hat{\eta}+(1-\hat{\eta}) \exp \left(\hat{\alpha}+\mathbf{x}_{i}^{\top} \hat{\beta}+\hat{\gamma} y\right)\right\} f\left(y \mid \mathbf{x}_{i}, \hat{\xi}\right) d y\right] \\
& =\frac{1}{n} \sum_{i=1}^{n} \frac{\int_{y} y\left\{\hat{\eta}+(1-\hat{\eta}) \exp \left(\hat{\alpha}+\mathbf{x}_{i}^{\top} \hat{\beta}+\hat{\gamma} y\right)\right\} f\left(y \mid \mathbf{x}_{i}, \hat{\xi}\right) d y}{\hat{\eta}+(1-\hat{\eta}) \exp \left\{t\left(\mathbf{x}_{i}, \hat{\theta}\right)\right\}}
\end{aligned}
$$

We use the normal model as an illustrating example: $f(y \mid \mathbf{x}, \xi)$ is chosen to be the density function of $N\left(\mu(\mathbf{x}, \xi), \sigma^{2}(\mathbf{x}, \xi)\right)$. In this example, the proposed mean estimator in (3.12) becomes

$$
\hat{\mu}=\frac{1}{n} \sum_{i=1}^{n} \frac{\hat{\eta} \hat{\mu}_{i}+(1-\hat{\eta})\left(\hat{\mu}_{i}+\hat{\gamma} \hat{\sigma}_{i}^{2}\right) \exp \left(\hat{\alpha}+\mathbf{x}_{i}^{\top} \hat{\beta}+\hat{\mu}_{i} \hat{\gamma}+0.5 \hat{\gamma}^{2} \hat{\sigma}_{i}^{2}\right)}{\hat{\eta}+(1-\hat{\eta}) \exp \left(\hat{\alpha}+\mathbf{x}_{i}^{\top} \hat{\beta}+\hat{\mu}_{i} \hat{\gamma}+0.5 \hat{\gamma}^{2} \hat{\sigma}_{i}^{2}\right)}
$$

where $\hat{\mu}_{i}=\mu\left(\mathbf{x}_{i}, \hat{\xi}\right)$ and $\hat{\sigma}_{i}^{2}=\sigma^{2}\left(\mathbf{x}_{i}, \hat{\xi}\right)$.

The next theorem establishes the asymptotic normality of the proposed estimator $\hat{\mu}$ in (3.12).

Theorem 2. Under the conditions of Theorem 1, as n goes to infinity, $\sqrt{n}(\hat{\mu}-\mu) \rightarrow N\left(0, \sigma^{2}\right)$ in distribution, where $\sigma^{2}=\operatorname{Var}\left\{K\left(\mathbf{X} ; \theta_{0}, \eta_{0}\right)\right\}+A^{\top} V^{-1} A$ with

$$
K(\mathbf{x} ; \theta, \eta)=\frac{\int y\left\{\eta+(1-\eta) \exp \left(\alpha+\mathbf{x}^{\top} \beta+\gamma y\right)\right\} f(y \mid \mathbf{x}, \xi) d y}{\eta+(1-\eta) \exp \left\{\alpha+\mathbf{x}^{\top} \beta+c(\mathbf{x}, \gamma, \xi)\right\}}
$$

and $A=\mathbb{E}\left\{\nabla_{\theta} K\left(\mathbf{X} ; \theta_{0}, \eta_{0}\right)\right\}$. 
When Wald-type intervals are constructed for $\mu$ based on Theorem 2, we need a consistent estimator of $\sigma^{2}$, which can be constructed based on consistent estimators of $A$, $\operatorname{Var}\left\{K\left(\mathbf{X} ; \theta_{0}, \eta_{0}\right)\right\}$, and $V$. Reasonable estimators for these three quantities are

$$
\begin{aligned}
\hat{A} & =n^{-1} \sum_{i=1}^{n} \nabla_{\theta} K\left(\mathbf{x}_{i} ; \hat{\theta}, \hat{\eta}\right), \\
\widehat{\operatorname{Var}}\left\{K\left(\mathbf{X} ; \theta_{0}, \eta_{0}\right)\right\} & =n^{-1} \sum_{i=1}^{n}\{K(\mathbf{X} ; \hat{\theta}, \hat{\eta})\}^{2}-\left\{n^{-1} \sum_{i=1}^{n} K(\mathbf{X} ; \hat{\theta}, \hat{\eta})\right\}^{2},
\end{aligned}
$$

and

$$
\hat{V}=n^{-1} \sum_{i=1}^{n}\left[\left\{1-\pi\left(\mathbf{x}_{i}, \hat{\theta}, \hat{\eta}\right)\right\} \pi\left(\mathbf{x}_{i}, \hat{\theta}, \hat{\eta}\right)\left\{\nabla_{\theta} t\left(\mathbf{x}_{i}, \hat{\theta}\right)\right\}^{\otimes 2}+d_{i} I_{e}\left\{\nabla_{\xi} f\left(y_{i} \mid \mathbf{x}_{i}, \hat{\xi}\right)\right\}^{\otimes 2} I_{e}^{\top}\right] .
$$

These estimators are consistent because $(\hat{\theta}, \hat{\eta})$ is consistent and $K$ is smooth in all its arguments. Consequently a consistent estimator of $\sigma^{2}$ is

$$
\hat{\sigma}^{2}=\widehat{\operatorname{Var}}\left\{K\left(\mathbf{X} ; \theta_{0}, \eta_{0}\right)\right\}+\hat{A}^{\top} \hat{V}^{-1} \hat{A} .
$$

\subsection{Semiparametric efficiency}

We make the same model assumptions as Riddles et al. (2016): the logistic model in (1.1) for the propensity score and a parametric model $f(y \mid \mathbf{x}, \xi)$ for $\operatorname{pr}(y \mid \mathbf{x}, D=1)$, and leave $\operatorname{pr}(\mathbf{x} \mid D=1)$ completely unspecified. Therefore our model setup is semi-parametric. Next we show that the estimators $(\hat{\theta}, \hat{\eta})$ and $\hat{\mu}$, which are built on the above semi-parametric model, are semiparametrically efficient.

Theorem 3. Under the conditions of Theorem 1, the MLEs $(\hat{\theta}, \hat{\eta})$ and $\hat{\mu}$ are both semiparametrically efficient in sense that their asymptotic variances attain the corresponding semiparametric efficiency lower bounds.

We make some comments on Theorem 3 and the results in Riddles et al. (2016), Morikawa and Kim (2016) and Ai et al. (2018). Note that Riddles et al. (2016)'s estimator was constructed under the same model assumptions as ours. Theorem 3 implies that the asymptotic variance of their mean estimator is no less than $\sigma^{2}$, the asymptotic variance of the MLE $\hat{\mu}$ and also the semiparametric efficiency lower bound for estimating $\mu$. 
When only the parametric propensity score assumption is made, Morikawa and Kim (2016) derived the semiparametric efficiency lower bound for the parameter of interest such as the response mean, and proposed two adaptive estimators whose asymptotic variances attain the lower bound. Ai et al. (2018) proposed an estimation method based on the generalized method of moments, and showed that as the number of moments increases appropriately their estimator also attains the lower bound of Morikawa and Kim (2016). According to Tsiatis (2006), the semiparametric efficiency lower bound is equal to the supremum of the asymptotic variances of the MLEs under all parametric submodels. Since the model assumptions in Morikawa and Kim (2016) is weaker than ours, the set of all parametric submodels considered in Morikawa and Kim (2016) contains all parametric submodels considered in this paper. Consequently, when the parameter of interest is the response mean, the semiparametric efficiency lower bound of Morikawa and Kim (2016) is no less than $\sigma^{2}$. Hence the asymptotic variances of Morikawa and Kim (2016)'s two adaptive estimators and Ai et al. (2018)'s estimator are no less than that of our estimator $\hat{\mu}$.

\subsection{Model checking}

Based on the completely observed data $\left\{\left(y_{i}, \mathbf{x}_{i}, d_{i}=1\right), i=1, \ldots, n_{1}\right\}$, we can directly examine the correctness of the model assumption $\operatorname{pr}(y \mid \mathbf{x}, D=1)=f(y \mid \mathbf{x}, \xi)$. One method for this is Ducharme and Ferrigno (2012)'s omnibus goodness-of-fit test for conditional distributions. Another question about the proposed model is the reliability of the parametric model assumption on the propensity score $\operatorname{pr}(D=1 \mid \mathbf{x}, y)$. Since we do not observe $y_{i}$ 's for $\left\{\left(\mathbf{x}_{i}, d_{i}=0\right), i=n_{1}+1, \ldots, n\right\}$, we do not have direct data to check this. However, the question can be answered indirectly by testing the goodness-of-fit of the DRM (2.6). The latter problem has been studied by many researchers and can be solved by the tests of Qin and Zhang (1997), Cheng and Chu (2004), Bondell (2007), and others. 


\section{Simulation}

\section{$4.1 \quad$ Set-up}

We carry out simulations to investigate the finite-sample performance of the proposed estimator for the population mean of the response. We compare the proposed mean estimator $\hat{\mu}$ with four others: (1) Morikawa and Kim (2016)'s adaptive estimator with correctly specified parametric form for $\operatorname{pr}(y \mid \mathbf{x}, D=1), \tilde{\mu}_{t}$, (2) Morikawa and Kim (2016)'s adaptive estimator without specifying a parametric form for $\operatorname{pr}(y \mid \mathbf{x}, D=1), \tilde{\mu}_{n p},(3)$ the sample mean of the observed response, $\bar{y}_{r}$, and (4) the sample mean of all the responses when there are no missing data, $\bar{y}$. When $\operatorname{pr}(y \mid \mathbf{x}, D=1)$ is correctly specified, Morikawa and Kim (2016) showed that $\tilde{\mu}_{t}$ is more efficient than Riddles et al. (2016)'s estimator, and further Ai et al. (2018)'s estimator has the same asymptotic variance as $\tilde{\mu}_{t}$. Hence Riddles et al. (2016)'s and Ai et al. (2018)'s methods are not included in the comparison.

We generate data from the following three examples.

Example 1. Let $\mathbf{x}=(z, u)^{\top}$, where $u$ is a Bernoulli random variable with success probability $0.5, z$ follows the uniform distribution on $(-1,1)$, and $u$ and $z$ are independent. We choose $\operatorname{pr}(D=1 \mid \mathbf{x}, y)=1 /\{1+\exp (-1.7-0.4 u+0.5 y)\}$ and set $\operatorname{pr}(y \mid \mathbf{x}, D=1)=f(y \mid \mathbf{x}, \xi)$ to the density function of $N\left(\mu(\mathbf{x}), \sigma^{2}\right)$, where $\mu(\mathbf{x})=\exp (0.5-u+1.5 z)$ and $\sigma^{2}=1$ or 4 .

Example 2. Let $\mathbf{x}=(z, u)^{\top}$, where $u \sim N(1,1), z \sim N(0,1)$ and $u$ and $z$ are independent. We choose $\operatorname{pr}(D=1 \mid \mathbf{x}, y)=1 /\{1+\exp (-1.7-0.4 u+0.5 y)\}$ and set $\operatorname{pr}(y \mid \mathbf{x}, D=1)=$ $f(y \mid \mathbf{x}, \xi)$ to the density function of $N\left(\mu(\mathbf{x}), \sigma^{2}\right)$, where $\mu(\mathbf{x})=2.5-u+1.5 z$ and $\sigma^{2}=1$ or 4 .

Example 3. The covariate $x$ follows $N(2,1)$. We choose $\operatorname{pr}(D=1 \mid x, y)=1 /\{1+\exp (-2.7-$ $0.4 x+0.5 y)\}$ and set $\operatorname{pr}(y \mid x, D=1)=f(y \mid x, \xi)$ to the density function of $N\left(\mu(x), \sigma^{2} e^{0.5 x}\right)$, where $\mu(x)=2-x+x^{2}$ and $\sigma^{2}=1$ or $e^{0.7}$.

Example 1 is Scenario 2 of Morikawa and Kim (2016) except that we consider $\sigma^{2}=1$ and 4, while Morikawa and Kim (2016) only considered $\sigma^{2}=1$. Example 2 represents the case where the mean function is a linear function of $\mathbf{x}$. Both Examples 11 and 2 have an instrument variable so the model parameters are identifiable. Example 3 represents the case that there is no instrument variable, but the model parameters are still identifiable. The 
true values of $\mu$ and the missing probability $1-\eta$ for the three examples are tabulated in Table 1 .

Table 1: True values of $\mu$ and the missing probability $1-\eta$ in Examples 13 .

\begin{tabular}{cccc|cccc}
\hline Example & $\sigma^{2}$ & $\mu$ & $1-\eta$ & Example & $\sigma^{2}$ & $\mu$ & $1-\eta$ \\
\hline 1 & 1 & 1.748 & 0.294 & 1 & 4 & 2.326 & 0.362 \\
2 & 1 & 1.638 & 0.275 & 2 & 4 & 2.177 & 0.339 \\
3 & 1 & 3.129 & 0.277 & 3 & $e^{0.7}$ & 3.289 & 0.299 \\
\hline
\end{tabular}

\subsection{Point estimation}

In this section, we evaluate the performance of the five mean estimators in terms of the relative bias (RB) and mean square error (MSE). We set $n=500$ and 2000 for all the three examples, and use 2000 for the number of repetitions in all our simulations. The simulation results are summarized in Table 2 .

It is worth mentioning that we encountered some numerical problems in the implementation of Morikawa and Kim (2016)'s adaptive estimator $\tilde{\mu}_{t}$, in Example 1 with $n=500,2000$ and $\sigma^{2}=4$, Example 2 with $n=500$ and $\sigma^{2}=4$, and Example 3 with $n=500$ and $\sigma^{2}=e^{0.7}$. Morikawa and Kim (2016)'s algorithm either does not converge or produces too big (greater than 5) or too small (less than 0) mean estimates. Throughout the simulation study, the performance of $\tilde{\mu}_{t}$ are evaluated based only on the estimates between 0 and 5 .

We first look at the results for Example 1. When $\sigma^{2}=1$, the relative biases of the proposed estimator and Morikawa and Kim (2016)'s two adaptive estimators are all small. The proposed estimator has slightly smaller mean square errors than Morikawa and Kim (2016)'s two adaptive estimators, whose mean square errors are quite close to each other. When $\sigma^{2}$ is increased to 4 , the relative biases of $\tilde{\mu}_{\eta p}$ become much bigger. The proposed estimator has much smaller mean square errors than Morikawa and Kim (2016)'s two adaptive estimators. The comparison between $\hat{\mu}$ and $\tilde{\mu}_{t}$ in Example 2 is similar to that for Example 1 . For Example 2, compared with $\tilde{\mu}_{t}, \tilde{\mu}_{n p}$ has much bigger relative biases and mean square errors especially for larger $\sigma^{2}$. Next, we examine the results for Example 3, in which there is no instrumental variable. The proposed estimator has small relative biases in all situations. Its mean square errors are significantly smaller than Morikawa and Kim (2016)'s two adaptive estimators. Finally, as expected, $\bar{y}_{r}$ has large relative biases and the largest mean square 
Table 2: Relative bias $(\mathrm{RB} ; \times 100)$ and mean square error $(\mathrm{MSE} ; \times 100)$ of five estimates of $\mu$.

\begin{tabular}{|c|c|c|c|c|c|c|c|c|c|c|c|}
\hline$n$ & & $\mu$ & $\tilde{\mu}_{t}$ & $\tilde{\mu}_{n p}$ & $\bar{y}_{r}$ & $\bar{y}$ & $\mu$ & $\tilde{\mu}_{t}$ & $\tilde{\mu}_{n p}$ & $\bar{y}_{r}$ & $\bar{y}$ \\
\hline & & & Exa & iple [1: & $=1$ & & \multicolumn{5}{|c|}{ Example [1] $\sigma^{2}=4$} \\
\hline 500 & RB & & -0 & -1.31 & -32.61 & -0.19 & & -1.18 & -8.45 & -51.71 & -0.19 \\
\hline 500 & MSE & & 0.98 & 1.04 & 33.10 & & & 7.17 & & 1 & \\
\hline 2000 & $\mathrm{RB}$ & & 0.04 & -0 & -32 & 0.03 & & -( & 21 & -51.57 & 1 \\
\hline 2000 & & & 0.24 & 0.24 & 32.49 & & & .24 & .91 & & \\
\hline & & \multicolumn{5}{|c|}{ Example 2: $\sigma^{2}=1$} & \multicolumn{5}{|c|}{ Example 2 $\sigma^{2}=4$} \\
\hline 5 & B & & -0.28 & -4.49 & -35.83 & 00 & & & & -56.27 & .05 \\
\hline & $\mathrm{SE}$ & & & & & & & & & 9 & \\
\hline 00 & & & 3 & -2.85 & -35 & & & 0.09 & -15.01 & 05 & 09 \\
\hline 2000 & MSE & & 0.27 & 0.48 & 34.43 & & & 1.40 & 11.43 & 149.48 & 17 \\
\hline & & \multicolumn{5}{|c|}{ Example [3: $\sigma^{2}=1$} & \multicolumn{5}{|c|}{ Example 3] $\sigma^{2}=e^{0.7}$} \\
\hline 5 & & & -0.24 & -2.29 & -18.70 & 0 & & -0.71 & -3.08 & -23.00 & .06 \\
\hline 500 & MSE & & 1.82 & 1.48 & 34.79 & & & 3.99 & 2.52 & 58.19 & .21 \\
\hline 2000 & & & -0.02 & -1.20 & -18.70 & -0.04 & 0.05 & -0.11 & -1.67 & -23.06 & 0.00 \\
\hline 2000 & MSE & 25 & 0.38 & 0.39 & 34.35 & 0.23 & 0.41 & 0.80 & 0.69 & 57.76 & 0.29 \\
\hline
\end{tabular}

errors in all examples, while the ideal estimator $\bar{y}$ has small relative biases and the smallest mean square errors in all situations. When $\sigma^{2}$ is small, the proposed estimator has almost the same performance as the ideal estimator $\bar{y}$, indicating that it is nearly optimal and can be hardly improved.

\subsection{Interval estimation}

This section is devoted to comparing the coverage of Wald confidence intervals based on $\hat{\mu}, \tilde{\mu}_{t}$, and $\bar{y}_{r}$. The nonparametric bootstrap method with 200 bootstrap samples is used to estimate the asymptotic variance for each of the three mean estimators. Although the variance estimator in (3.14) can be used in the Wald-type confidence intervals based on $\hat{\mu}$, its complicated form makes it more difficult to calculate than bootstrap variance estimate. The bootstrap method is quite computationally intensive for $\tilde{\mu}_{n p}$. For example, in Example 1, it takes around 9 minutes and 2 hours respectively to calculate the bootstrap variances for $\tilde{\mu}_{n p}$ for a single replication when $n=500$ and $n=2000$. Hence we do not include it for comparison. Again the number of repetitions is 2000 in all cases. The simulation results are summarized in Table 3 ,

In most cases, both Wald confidence intervals based on $\hat{\mu}$ and $\tilde{\mu}_{t}$ have very close and 
accurate coverage probabilities. The exceptions are Example 1 and Example 3 with the smaller sample size $n=500$. For Example 1, both intervals have slight under coverage, while for Example 3, the Wald confidence interval based on $\tilde{\mu}_{t}$ has much over-coverage, in particular when $\sigma^{2}$ is large. When the sample size $n$ is increased to 2000, both intervals have perfect coverage accuracy. It is worth noting that for the Wald confidence interval based on $\tilde{\mu}_{t}$, the results with $\tilde{\mu}_{t}$ outside $[0,5]$ or not convergent were not taken into consideration. In all cases, the Wald confidence interval based on $\bar{y}_{r}$ has unacceptable coverage accuracy, which is most probably caused by the severe bias of $\bar{y}_{r}$. Overall the Wald confidence interval based on the proposed estimator $\hat{\mu}$ is the most accurate and desirable among the three interval estimators under comparison.

Table 3: Simulated coverage probabilities (\%) of bootstrap Wald-type confidence intervals based on $\hat{\mu}, \tilde{\mu}_{t}$, and $\bar{y}_{r}$ in Examples 13 .

\begin{tabular}{clllll|clllll}
\hline Example & $\sigma^{2}$ & $n$ & $\hat{\mu}$ & $\tilde{\mu}_{t}$ & $\bar{y}_{r}$ & Example & $\sigma^{2}$ & $n$ & $\hat{\mu}$ & $\tilde{\mu}_{t}$ & $\bar{y}_{r}$ \\
\hline 1 & 1 & 500 & 93.6 & 94.4 & 0 & 1 & 4 & 500 & 95.1 & 95.7 & 1.0 \\
1 & 1 & 2000 & 95.3 & 95.1 & 0 & 1 & 4 & 2000 & 94.7 & 94.2 & 0 \\
2 & 1 & 500 & 94.5 & 94.7 & 0.1 & 2 & 4 & 500 & 95.2 & 95.3 & 0 \\
2 & 1 & 2000 & 95.1 & 95.2 & 0 & 2 & 4 & 2000 & 95.4 & 95.2 & 0 \\
3 & 1 & 500 & 94.9 & 96.0 & 0 & 3 & $e^{0.7}$ & 500 & 95.7 & 97.5 & 0 \\
3 & 1 & 2000 & 95.0 & 94.7 & 0 & 3 & $e^{0.7}$ & 2000 & 94.8 & 95.5 & 0 \\
\hline
\end{tabular}

\section{An application}

We apply the proposed method to analyze the Korean Labor and Income Panel Study (KLIPS) data set (Kim and Yu,,2011; Wang et al., 2014; Shao and Wang, 2016; Morikawa and Kim, 2016), which includes $n=2506$ regular wage earners. We take the monthly income in 2006 as the response $y$, and use as covariates the monthly income in $2005\left(x_{1}\right)$, age $\left(x_{2}\right)$, education level $\left(x_{3}\right)$, and gender $\left(x_{4}\right)$. The variable $y$ has approximately $35 \%$ missing values, while all the covariate values are observed. The only outlier value of the education level, 99, is replaced by 6 , which is the nearest integer to the observed mean of the response $y$. We treat all the variables as continuous and suppose $\operatorname{pr}(D=0 \mid \mathbf{x}, y)=1 /\left\{1+\exp \left(\alpha^{*}+\mathbf{x}^{\top} \beta+y \gamma\right)\right\}$ and that $f(y \mid \mathbf{x}, \xi)$ is the density function of $N\left(\mu(\mathbf{x}, \xi), \sigma^{2}(\mathbf{x}, \xi)\right)$. We consider the following four scenarios of $\mathbf{x}, \mu(\mathbf{x}, \xi)$ and $\sigma^{2}(\mathbf{x}, \xi)$ : 
Model (1a): We take $x=x_{1}$ and assume $\mu(x, \xi)=\xi_{0}+\xi_{1} x+\xi_{2} x^{2}$ and $\sigma^{2}(x, \xi)=\exp \left(\xi_{3}+\right.$ $\left.\xi_{4} x\right)$.

Model (1b): We take $\mathbf{x}=\left(x_{1}, x_{2}\right)^{\top}$ and assume $\mu(\mathbf{x}, \xi)=\xi_{0}+\xi_{1} x_{1}+\xi_{2} x_{2}+\xi_{3} x_{1}^{2}+\xi_{4} x_{1} x_{2}+\xi_{5} x_{2}^{2}$ and $\sigma^{2}(\mathbf{x}, \xi)=\exp \left(\xi_{6}+\xi_{7} x_{6}+\xi_{8} x_{7}\right)$.

Model (1c): We take $\mathbf{x}=\left(x_{1}, x_{2}, x_{3}\right)^{\top}$ and assume $\mu(\mathbf{x}, \xi)=\xi_{0}+\xi_{1} x_{1}+\xi_{2} x_{2}+\xi_{3} x_{3}+\xi_{4} x_{1}^{2}+$ $\xi_{5} x_{1} x_{2}+\xi_{6} x_{1} x_{3}+\xi_{7} x_{2}^{2}+\xi_{8} x_{2} x_{3}+\xi_{9} x_{3}^{2}$ and $\sigma^{2}(\mathbf{x}, \xi)=\exp \left(\xi_{10}+\xi_{11} x_{1}+\xi_{12} x_{2}+\xi_{13} x_{3}\right)$.

Model (1d): We take $\mathbf{x}=\left(x_{1}, x_{2}, x_{3}, x_{4}\right)^{\top}$ and assume $\mu(\mathbf{x}, \xi)=\xi_{0}+\xi_{1} x_{1}+\xi_{2} x_{2}+\xi_{3} x_{3}+$ $\xi_{4} x_{4}+\xi_{5} x_{1}^{2}+\xi_{6} x_{1} x_{2}+\xi_{7} x_{1} x_{3}+\xi_{8} x_{2}^{2}+\xi_{9} x_{2} x_{3}+\xi_{10} x_{3}^{2}$ and $\sigma^{2}(\mathbf{x}, \xi)=\exp \left(\xi_{11}+\xi_{12} x_{1}+\right.$ $\left.\xi_{13} x_{2}+\xi_{14} x_{3}+\xi_{15} x_{4}\right)$.

Table 4 presents $\hat{\mu}, \tilde{\mu}_{t}$, and $\bar{y}_{r}$, and the corresponding Wald-type interval estimates at the 95\% confidence level. Their standard errors are estimated by the nonparametric bootstrap method with 200 bootstrap samples. The proposed mean estimates are all around 188 and the corresponding interval estimates are around [177, 197]. In comparison, Morikawa and Kim (2016)'s point and interval estimates are around 190 and [183, 197], except for model (1d), where the interval estimate $[174,205]$ is much wider. Meanwhile we have observed that Morikawa and Kim (2016)'s method suffers from numerical problems. Under the four models, their algorithm did not converge in $6.5 \%, 28.5 \%, 32.5 \%$, and $30 \%$, respectively, of the 200 bootstrap repetitions. In the data-set under study, the observations for the response $y$ are actually available, but made missing by Kim and Yu (2011) in their empirical study. The true mean of $y$ is 185.04 , which is trustable to be close to the population mean. The above observations indicate that the proposed method has less bias in point estimation and more stable performance in interval estimation than Morikawa and Kim (2016)'s method. Again the complete-case estimate $\bar{y}_{r}$ is unacceptably biased.

Based on the proposed full log-likelihood, the Bayesian Information Criteria (BIC) of the four models are 5393.443, 5375.292, 5336.675, and 5271.354, respectively, which suggests model (1d) is the best. We would recommend the use of model (1d) and estimate the population mean by 187.76 , which is the closest to the ideal sample mean 185.04 among all estimates. 
Table 4: Point and interval estimates of the response mean for the KLIPS data.

\begin{tabular}{cccc}
\hline \multicolumn{4}{c}{ KLIPS data } \\
Model & Method & Estimate & Interval Estimate \\
\hline \multirow{2}{*}{ 1a } & $\bar{y}_{r}$ & 205.71 & {$[200.12,211.29]$} \\
& $\hat{\mu}$ & 189.32 & {$[180.68,197.95]$} \\
& $\tilde{\mu}_{t}$ & 190.25 & {$[183.79,196.71]$} \\
& $\hat{\mu}$ & 189.15 & {$[180.78,197.52]$} \\
& $\tilde{\mu}_{t}$ & 190.16 & {$[181.90,198.43]$} \\
1c & $\hat{\mu}$ & 187.88 & {$[178.90,196.86]$} \\
& $\tilde{\mu}_{t}$ & 191.38 & {$[184.51,198.25]$} \\
1d & $\hat{\mu}$ & 187.76 & {$[177.28,198.25]$} \\
& $\tilde{\mu}_{t}$ & 189.61 & {$[171.10,208.12]$} \\
\hline
\end{tabular}

\section{Discussion}

In the development of our estimation procedure, we assume that the propensity score follows a logistic regression model only for convenience, since the logistic regression model has a close relationship with Anderson (1979)'s DRM. Our estimation procedure for the propensity score works for any parametric model provided it is identifiable.

\section{Appendix 1: Regularity conditions for $f(y \mid \mathbf{x}, \xi)$}

The following regularity conditions on $f(y \mid \mathbf{x}, \xi)$ mimic those for the consistency and asymptotic normality of the MLE under a regular parametric model on pp. 144-145 of Serfling (1980).

(A1) In a neighbourhood of $\xi_{0}, \log \{f(y \mid \mathbf{x}, \xi)\}$ is three-times differentiable with respect to $\xi$ for any $(y, \mathbf{x})$.

(A2) For $(\gamma, \xi)$ in a neighbourhood of $\left(\gamma_{0}, \xi_{0}\right)$ and any $\mathbf{x}$ on S, the inequality $\int e^{y \gamma} f(y \mid \mathbf{x}, \xi) d y<$ $\infty$ holds.

(A3) The matrix $V$ defined in (3.11) is well defined and nonsingular.

(A4) There exists a function $M(\mathbf{x})$ not depending on $(\gamma, \xi)$ such that $\mathbb{E}\{M(\mathbf{X})\}<\infty$ and $\left\|\int e^{y \gamma} \nabla_{\xi} f(y \mid \mathbf{x}, \xi) d y\right\|+\left\|\int e^{y \gamma} \nabla_{\xi, \xi} f(y \mid \mathbf{x}, \xi) d y\right\|+\left\|\int e^{y \gamma} \nabla_{\xi, \xi, \xi} f(y \mid \mathbf{x}, \xi) d y\right\|<M(\mathbf{x})$ 
uniformly for $(\gamma, \xi)$ in a neighbourhood of $\left(\gamma_{0}, \xi_{0}\right)$ and a neighbourhood of $\left(0, \xi_{0}\right)$. Here $\nabla_{\xi} f(y \mid \mathbf{x}, \xi), \nabla_{\xi, \xi} f(y \mid \mathbf{x}, \xi)$, and $\nabla_{\xi, \xi, \xi} f(y \mid \mathbf{x}, \xi)$ are the first, second, and third derivatives of $f(y \mid \mathbf{x}, \xi)$ with respect to $\xi$.

Conditions A1 and A2 guarantee that the first partial derivatives of $\ell_{2}(\alpha, \beta, \xi, \gamma)$ are well defined for any data. Conditions $\mathrm{A} 3$ and $\mathrm{A} 4$ ensure that $\ell_{2}(\alpha, \beta, \xi, \gamma)$ can be approximated by its second-order Taylor expansion at $\left(\alpha_{0}, \beta_{0}, \xi_{0}, \gamma_{0}\right)$ when $(\alpha, \beta, \xi, \gamma)$ lies in a neighbourhood of $\left(\alpha_{0}, \beta_{0}, \xi_{0}, \gamma_{0}\right)$.

\section{Appendix 2: Proof of Corollary 1}

We first consider Part (a). Since $z$ is an instrument variable, (2.6) becomes

$$
\operatorname{pr}(\mathbf{x} \mid D=0)=\exp \left\{\alpha+u^{\top} \beta+c(\mathbf{x}, \gamma, \xi)\right\} \operatorname{pr}(\mathbf{x} \mid D=1)
$$

The identification of $(\alpha, \beta, \gamma)$ is equivalent to the identification of $(\alpha, \beta, \gamma)$ in $\alpha+u^{\top} \beta+$ $c(\mathbf{x}, \gamma, \xi)$.

Recall that $f(y \mid \mathbf{x}, \xi)=\operatorname{pr}(y \mid \mathbf{x}, D=1)$. Then

$$
\begin{aligned}
f(y \mid \mathbf{x}, \xi) & =\operatorname{pr}(y \mid \mathbf{x}) \operatorname{pr}(D=1 \mid \mathbf{x}, y) / \int \operatorname{pr}(y \mid \mathbf{x}) \operatorname{pr}(D=1 \mid \mathbf{x}, y) d y \\
& =\operatorname{pr}(y \mid z, u) \operatorname{pr}(D=1 \mid u, y) / \int \operatorname{pr}(y \mid z, u) \operatorname{pr}(D=1 \mid u, y) d y
\end{aligned}
$$

Since $z$ is an instrument variable, it follows that $f(y \mid \mathbf{x}, \xi)$ must depend on $z$, and so must $c(\mathbf{x}, \gamma, \xi)$. Suppose $\left(\alpha_{1}, \beta_{1}, \gamma_{1}\right)$ and $\left(\alpha_{2}, \beta_{2}, \gamma_{2}\right)$ satisfy

$$
\alpha_{1}+u^{\top} \beta_{1}+c\left(\mathbf{x}, \gamma_{1}, \xi\right)=\alpha_{2}+u^{\top} \beta_{2}+c\left(\mathbf{x}, \gamma_{2}, \xi\right)
$$

for all $\mathbf{x}$, which implies

$$
c\left(\mathbf{x}, \gamma_{1}, \xi_{0}\right)-c\left(\mathbf{x}, \gamma_{2}, \xi_{0}\right)=\left(\alpha_{2}-\alpha_{1}\right)+u^{\top}\left(\beta_{2}-\beta_{1}\right)
$$

Since the left-hand side depends on $z$, while the right-hand side does not, we must have $\gamma_{1}=\gamma_{2}$, which further implies that $\alpha_{1}=\alpha_{2}$ and $\beta_{1}=\beta_{2}$. This indicates that the parameters $(\alpha, \beta, \gamma)$ are identifiable, which completes the proof of Part (a). 
We next consider Part (b). Suppose $\left(\alpha_{1}, \beta_{1}, \gamma_{1}\right)$ and $\left(\alpha_{2}, \beta_{2}, \gamma_{2}\right)$ satisfy

$$
\alpha_{1}+\beta_{1}^{\top} \mathbf{x}+c\left(\mathbf{x}, \gamma_{1}, \xi_{0}\right)=\alpha_{2}+\beta_{2}^{\top} \mathbf{x}+c\left(\mathbf{x}, \gamma_{2}, \xi_{0}\right)
$$

for all $\mathbf{x} \in S$. According to the expression for $c\left(\mathbf{x}, \gamma, \xi_{0}\right)$, this implies that

$$
\begin{aligned}
& \sum_{i=1}^{k} a_{i}\left(\gamma_{1}\right) g_{i}(\mathbf{x})+\left\{\alpha_{1}+a_{k+1}\left(\gamma_{1}\right)\right\}+\mathbf{x}^{\top}\left\{\beta_{1}+a_{k+2}\left(\gamma_{1}\right)\right\} \\
= & \sum_{i=1}^{k} a_{i}\left(\gamma_{2}\right) g_{i}(\mathbf{x})+\left\{\alpha_{2}+a_{k+1}\left(\gamma_{2}\right)\right\}+\mathbf{x}^{\top}\left\{\beta_{2}+a_{k+2}\left(\gamma_{2}\right)\right\} .
\end{aligned}
$$

Since $1, \mathbf{x}, g_{1}(\mathbf{x}), \ldots, g_{k}(\mathbf{x})$ are linearly independent, it follows that

$$
\begin{aligned}
\left(a_{1}\left(\gamma_{1}\right), \ldots, a_{k}\left(\gamma_{1}\right)\right) & =\left(a_{1}\left(\gamma_{2}\right), \ldots, a_{k}\left(\gamma_{2}\right)\right) \\
\alpha_{1}+a_{k+1}\left(\gamma_{1}\right) & =\alpha_{2}+a_{k+1}\left(\gamma_{2}\right) \\
\beta_{1}+a_{k+2}\left(\gamma_{1}\right) & =\beta_{2}+a_{k+2}\left(\gamma_{2}\right)
\end{aligned}
$$

hold simultaneously. Because $\left(a_{1}\left(\gamma_{1}\right), \ldots, a_{k}\left(\gamma_{1}\right)\right) \neq\left(a_{1}\left(\gamma_{2}\right), \ldots, a_{k}\left(\gamma_{2}\right)\right)$ for any $\gamma_{1} \neq \gamma_{2}$, the first equation implies $\gamma_{1}=\gamma_{2}$. Then the last two equations lead to $\alpha_{1}=\alpha_{2}$ and $\beta_{1}=\beta_{2}$. This completes the proof of Part (b) and that of Corollary 1.

\section{Appendix 3: Preparation for proving Theorems 1-3}

\section{Re-expression}

It can be verified that

$$
\ell_{2}(\theta)=h\left(\theta, \lambda_{\theta}\right)
$$

where

$$
\begin{aligned}
h(\theta, \lambda)= & \sum_{i=1}^{n_{1}} \log \left\{f\left(y_{i} \mid \mathbf{x}_{i}, \xi\right)\right\}+\sum_{i=n_{1}+1}^{n} t\left(\mathbf{x}_{i}, \theta\right) \\
& -\sum_{i=1}^{n} \log \left\{1+\lambda\left[\exp \left\{t\left(\mathbf{x}_{i}, \theta\right)\right\}-1\right]\right\}
\end{aligned}
$$

and $\lambda_{\theta}$ is the solution to $\nabla_{\lambda} h=0$. 
Let $\hat{\lambda}$ be the solution to (3.9) with $\hat{\theta}$ in place of $\theta$. We first discuss some properties of $\hat{\lambda}$. It can be verified that $(\hat{\theta}, \hat{\lambda})$ satisfy

$$
\nabla_{\alpha} h(\hat{\theta}, \hat{\lambda})=0, \quad \nabla_{\lambda} h(\hat{\theta}, \hat{\lambda})=0
$$

Note that

$$
\begin{aligned}
& \nabla_{\lambda} h(\theta, \lambda)=-\sum_{i=1}^{n} \frac{\exp \left\{t\left(\mathbf{x}_{i}, \theta\right)\right\}-1}{1+\lambda\left[\exp \left\{t\left(\mathbf{x}_{i}, \theta\right)\right\}-1\right]}=0 \quad \text { and } \\
& \nabla_{\alpha} h(\theta, \lambda)=n_{2}-\lambda \sum_{i=1}^{n} \frac{\exp \left\{t\left(\mathbf{x}_{i}, \theta\right)\right\}}{1+\lambda\left[\exp \left\{t\left(\mathbf{x}_{i}, \theta\right)\right\}-1\right]}=0
\end{aligned}
$$

together imply that

$$
\hat{\lambda}=n_{2} / n
$$

which converges in probability to $\lambda_{0}=1-\eta_{0}$.

For convenience of presentation, let $\omega=\left(\theta^{\top}, \lambda\right)^{\top}$. It can be verified that $\hat{\omega}=\left(\hat{\theta}^{\top}, \hat{\lambda}\right)^{\top}$ is the solution to $\partial h(\theta, \lambda) / \partial \omega=0$. To investigate the asymptotic properties of $(\hat{\theta}, \hat{\lambda})$, we need their approximations, which can be obtained via the second-order Taylor expansion of $h(\theta, \lambda)$ around $\omega=\omega_{0} \equiv\left(\theta_{0}^{\top}, \lambda_{0}\right)^{\top}$. In the next subsection, we derive the forms of $\partial h\left(\theta_{0}, \lambda_{0}\right) / \partial \omega$ and $\partial^{2} h\left(\theta_{0}, \lambda_{0}\right) /\left(\partial \omega \partial \omega^{\top}\right)$ and study their properties.

\section{First and second derivatives of $h(\theta, \lambda)$ at $\omega=\omega_{0}$}

For convenience of presentation, we write $\pi_{i}=\pi\left(\mathbf{x}_{i}\right)$. Denote

$$
u_{n}=\left(u_{n 1}, u_{n 2}^{\top}\right)^{\top}
$$

where

$$
\begin{aligned}
& u_{n 1}=\nabla_{\theta} h\left(\theta_{0}, \lambda_{0}\right)=\sum_{i=1}^{n}\left[\left(1-d_{i}-\pi_{i}\right) \nabla_{\theta} t\left(\mathbf{x}_{i}, \theta_{0}\right)+d_{i} I_{e} \nabla_{\xi} \log \left\{f\left(y_{i} \mid \mathbf{x}_{i}, \xi_{0}\right)\right\}\right], \\
& u_{n 2}=\nabla_{\lambda} h\left(\theta_{0}, \lambda_{0}\right)=\frac{1}{\lambda_{0}\left(1-\lambda_{0}\right)} \sum_{i=1}^{n}\left(\lambda_{0}-\pi_{i}\right) .
\end{aligned}
$$

After some calculation, it can be verified that the second derivatives of $h(\theta, \lambda)$ at $\left(\theta_{0}, \lambda_{0}\right)$ 
are

$$
\begin{aligned}
\nabla_{\theta \theta^{\top}} h\left(\theta_{0}, \lambda_{0}\right)= & V_{n}=\sum_{i=1}^{n} d_{i} I_{e} \nabla_{\xi \xi} \log \left\{f\left(y_{i} \mid \mathbf{x}_{i}, \xi_{0}\right)\right\} I_{e}^{\top} \\
& +\sum_{i=1}^{n}\left(1-d_{i}-\pi_{i}\right) \nabla_{\theta \theta} t\left(\mathbf{x}_{i}, \theta_{0}\right) \\
& -\sum_{i=1}^{n} \pi_{i}\left(1-\pi_{i}\right)\left\{\nabla_{\theta} t\left(\mathbf{x}_{i}, \theta_{0}\right)\right\}^{\otimes 2}, \\
\nabla_{\theta \lambda} h\left(\theta_{0}, \lambda_{0}\right)= & \frac{1}{\lambda_{0}\left(1-\lambda_{0}\right)} V_{n} \mathbf{e}_{1}, \\
\nabla_{\lambda \lambda} h\left(\theta_{0}, \lambda_{0}\right)= & \frac{1}{\lambda_{0}^{2}\left(1-\lambda_{0}\right)^{2}} \sum_{i=1}^{n}\left(\lambda_{0}-\pi_{i}\right)^{2} .
\end{aligned}
$$

\section{Some useful technical lemmas}

When deriving the asymptotic distribution of $\hat{\theta}$, we need to use $\mathbb{E}\left\{\nabla_{\theta \theta^{\top}} h\left(\theta_{0}, \lambda_{0}\right)\right\}, \mathbb{E}\left\{\nabla_{\theta \lambda} h\left(\theta_{0}, \lambda_{0}\right)\right\}$, $\mathbb{E}\left\{\nabla_{\lambda \lambda} h\left(\theta_{0}, \lambda_{0}\right)\right\}$, and the expectation and variance of $u_{n}$ defined in (A.3). We need the following lemma to simplify our calculation.

Lemma 1. The following equations hold:

$$
\begin{aligned}
\mathbb{E}\left[d_{i} \nabla_{\xi} \log \left\{f\left(y_{i} \mid \mathbf{x}_{i}, \xi_{0}\right)\right\}\right] & =0, \\
\mathbb{E}\left[d_{i} \nabla_{\xi \xi} \log \left\{f\left(y_{i} \mid \mathbf{x}_{i}, \xi_{0}\right)\right\}\right] & =-\mathbb{E}\left\{d_{i}\left[\nabla_{\xi} \log \left\{f\left(y_{i} \mid \mathbf{x}_{i}, \xi_{0}\right)\right\}\right]^{\otimes 2}\right\}, \\
-\frac{1}{n} \mathbb{E}\left\{\nabla_{\theta \theta^{\top}} h\left(\theta_{0}, \lambda_{0}\right)\right\} & =V, \\
-\frac{1}{n} \mathbb{E}\left\{\nabla_{\theta \lambda} h\left(\theta_{0}, \lambda_{0}\right)\right\} & =\frac{1}{\lambda_{0}\left(1-\lambda_{0}\right)} V \mathbf{e}_{1}, \\
-\frac{1}{n} \mathbb{E}\left\{\nabla_{\lambda \lambda} h\left(\theta_{0}, \lambda_{0}\right)\right\} & =\frac{\mathbf{e}_{1}^{\top} V \mathbf{e}_{1}-\lambda_{0}\left(1-\lambda_{0}\right)}{\lambda_{0}^{2}\left(1-\lambda_{0}\right)^{2}} .
\end{aligned}
$$

Proof. By the fact $f(y \mid \mathbf{x}, \xi)=\operatorname{pr}(Y=y \mid \mathbf{X}=\mathbf{x}, D=1)$, it can be verified that

$$
\begin{aligned}
\mathbb{E}\left[\nabla_{\xi} \log \left\{f\left(y_{i} \mid \mathbf{x}_{i}, \xi_{0}\right)\right\} \mid \mathbf{x}_{i}, d_{i}=1\right] & =0 \\
\mathbb{E}\left[\nabla_{\xi \xi} \log \left\{f\left(y_{i} \mid \mathbf{x}_{i}, \xi_{0}\right)\right\} \mid \mathbf{x}_{i}, d_{i}=1\right] & =-\mathbb{E}\left\{\left[\nabla_{\xi} \log \left\{f\left(y_{i} \mid \mathbf{x}_{i}, \xi_{0}\right)\right\}\right]^{\otimes 2} \mid \mathbf{x}_{i}, d_{i}=1\right\}
\end{aligned}
$$

which imply respectively Equations (A.4) and (A.5) by conditioning on $\left(\mathbf{x}_{i}, d_{i}=1\right)$.

Equations (A.6) and (A.7) follows immediately from (A.5). To prove (A.8), by noticing

$$
\lambda_{0}=1-\eta_{0}=\operatorname{pr}(D=0) \text { and } \pi(\mathbf{x})=\operatorname{pr}(D=0 \mid \mathbf{x})
$$


we have $\mathbb{E}\{\pi(\mathbf{X})\}=\lambda_{0}$ and

$$
\frac{1}{n} \mathbb{E}\left\{\nabla_{\lambda \lambda} h\left(\theta_{0}, \lambda_{0}\right)\right\}=\mathbb{E}\left\{\lambda_{0}-\pi\left(\mathbf{x}_{i}\right)\right\}^{2}=\mathbb{E}\left[\{\pi(\mathbf{X})\}^{2}\right]-\lambda_{0}^{2} .
$$

Since $\mathbf{e}_{1}^{\top} V \mathbf{e}_{1}=\mathbb{E}[\pi(\mathbf{X})\{1-\pi(\mathbf{X})\}]=-\mathbb{E}\left[\{\pi(\mathbf{X})\}^{2}\right]+\lambda_{0}$, Equation (A.8) follows by comparing this equation with (A.9). This finishes the proof.

The final lemma presents the expectation and variance of $u_{n}$.

Lemma 2. With $u_{n}$ defined in (A.3), we have $\mathbb{E}\left(u_{n}\right)=0$ and

$$
\frac{1}{n} \operatorname{Var}\left(u_{n}\right)=U=\left(\begin{array}{cc}
V & 0 \\
0 & \frac{-\mathbf{e}_{1}^{\top} V \mathbf{e}_{1}+\lambda_{0}\left(1-\lambda_{0}\right)}{\lambda_{0}^{2}\left(1-\lambda_{0}\right)^{2}}
\end{array}\right) .
$$

Proof. The result $\mathbb{E}\left(u_{n 2}\right)=0$ follows from $\pi(\mathbf{x})=\operatorname{pr}(D=0 \mid \mathbf{X}=\mathbf{x})$ and Equation (A.4).

For $\operatorname{Var}\left(u_{n}\right)$, we first calculate $\operatorname{Var}\left(u_{n 2}\right)$. It can be seen that

$$
\frac{1}{n} \operatorname{Var}\left(u_{n 2}\right)=\frac{1}{\lambda_{0}^{2}\left(1-\lambda_{0}\right)^{2}} \mathbb{E}\left[\left\{\lambda_{0}-\pi\left(\mathbf{x}_{i}\right)\right\}^{2}\right]=\frac{1}{\lambda_{0}^{2}\left(1-\lambda_{0}\right)^{2}}\left[\mathbb{E}\left\{\pi\left(\mathbf{x}_{i}\right)\right\}^{2}-\lambda_{0}^{2}\right] .
$$

We have shown that $\mathbf{e}_{1}^{\top} V \mathbf{e}_{1}=-\mathbb{E}\left[\{\pi(\mathbf{X})\}^{2}\right]+\lambda_{0}$ in the proof of Lemma 1 . Therefore

$$
\frac{1}{n} \operatorname{Var}\left(u_{n 2}\right)=\frac{-\mathbf{e}_{1}^{\top} V \mathbf{e}_{1}+\lambda_{0}\left(1-\lambda_{0}\right)}{\lambda_{0}^{2}\left(1-\lambda_{0}\right)^{2}} .
$$

It remains to calculate $\operatorname{Var}\left(u_{n 1}\right)$. Re-write

$$
u_{n 1}=\sum_{i=1}^{n}\left(u_{n 11, i}+u_{n 12, i}\right)
$$

where

$$
\begin{aligned}
& u_{n 11, i}=\left(1-d_{i}-\pi_{i}\right) \nabla_{\theta} t\left(\mathbf{x}_{i}, \theta_{0}\right) \\
& u_{n 12, i}=d_{i} I_{e} \nabla_{\xi} \log \left\{f\left(y_{i} \mid \mathbf{x}_{i}, \xi_{0}\right)\right\} .
\end{aligned}
$$

Since both $u_{n 11, i}$ and $u_{n 12, i}$ have mean zero, it follows from equality (A.4) that

$$
\frac{1}{n} \operatorname{Var}\left(u_{n 1}\right)=\mathbb{E}\left\{\left(u_{n 11, i}+u_{n 12, i}\right)^{\otimes 2}\right\}=\mathbb{E}\left(u_{n 11, i}^{\otimes 2}\right)+\mathbb{E}\left(u_{n 12, i}^{\otimes 2}\right) .
$$

Because $\mathbb{E}\left(d_{i}=0 \mid \mathbf{x}_{i}\right)=\operatorname{pr}\left(D=0 \mid \mathbf{X}=\mathbf{x}_{i}\right)=\pi\left(\mathbf{x}_{i}\right)$, by conditioning on $\mathbf{x}_{i}$, we have

$$
\begin{aligned}
\mathbb{E}\left(u_{n 11, i}^{\otimes 2}\right) & =\mathbb{E}\left[\left\{\left(1-d_{i}-\pi_{i}\right) \nabla_{\theta} t\left(\mathbf{x}_{i}, \theta_{0}\right)\right\}^{\otimes 2}\right] \\
& =\mathbb{E}\left[\left(\pi\left(\mathbf{x}_{i}\right)\left\{1-\pi\left(\mathbf{x}_{i}\right)\right\}\left\{\nabla_{\theta} t\left(\mathbf{x}_{i}, \theta_{0}\right)\right\}^{\otimes 2}\right]\right.
\end{aligned}
$$

Clearly $\mathbb{E}\left(u_{n 12, i}^{2}\right)=\mathbb{E}\left[d_{i} I_{e} \nabla_{\xi} \log \left\{f\left(y_{i} \mid \mathbf{x}_{i}, \xi_{0}\right)\right\}\right]^{2}$. This proves $\frac{1}{n} \mathbb{V} \operatorname{ar}\left(u_{n 1}\right)=V$ by comparing the expression of $V$ with $\frac{1}{n} \operatorname{Var}\left(u_{n 1}\right)$. 


\section{Appendix 4: Proof of Theorem 1}

We start with Part (a). Using a similar argument to that used in the proofs of Lemma 1 and Theorem 1 of Qin and Lawless (1994), we have $\hat{\theta}=\theta_{0}+O_{p}\left(n^{-1 / 2}\right)$ and $\hat{\lambda}-\lambda_{0}=O_{p}\left(n^{-1 / 2}\right)$.

Next we investigate the asymptotic approximation of $\hat{\theta}$.

The maximum likelihood estimator $\hat{\theta}$ of $\theta$ and the associated Lagrange multiplier $\hat{\lambda}$ must satisfy

$$
\left(\begin{array}{c}
\nabla_{\theta} h(\hat{\theta}, \hat{\lambda}) \\
\nabla_{\lambda} h(\hat{\theta}, \hat{\lambda})
\end{array}\right)=0
$$

Applying a first-order expansion to the left-hand side of the above equation gives

$$
0=\left(\begin{array}{c}
\nabla_{\theta} h\left(\theta_{0}, \lambda_{0}\right) \\
\nabla_{\lambda} h\left(\theta_{0}, \lambda_{0}\right)
\end{array}\right)+\left(\begin{array}{cc}
\nabla_{\theta \theta^{\top}} h\left(\theta_{0}, \lambda_{0}\right) & \nabla_{\theta \lambda} h\left(\theta_{0}, \lambda_{0}\right) \\
\nabla_{\lambda \theta^{\top}} h\left(\theta_{0}, \lambda_{0}\right) & \nabla_{\lambda \lambda} h\left(\theta_{0}, \lambda_{0}\right)
\end{array}\right)\left(\begin{array}{c}
\hat{\theta}-\theta_{0} \\
\hat{\lambda}-\lambda_{0}
\end{array}\right)+o_{p}\left(n^{1 / 2}\right) .
$$

By Lemma 1,

$$
\left(\begin{array}{cc}
\nabla_{\theta \theta^{\top}} h\left(\theta_{0}, \lambda_{0}\right) & \nabla_{\theta \lambda} h\left(\theta_{0}, \lambda_{0}\right) \\
\nabla_{\lambda \theta^{\top}} h\left(\theta_{0}, \lambda_{0}\right) & \nabla_{\lambda \lambda} h\left(\theta_{0}, \lambda_{0}\right)
\end{array}\right)=-n W+o_{p}(n)
$$

where

$$
W=\left(\begin{array}{cc}
V & \frac{1}{\lambda_{0}\left(1-\lambda_{0}\right)} V \mathbf{e}_{1} \\
\frac{1}{\lambda_{0}\left(1-\lambda_{0}\right)} \mathbf{e}_{1}^{\top} V & \frac{\mathbf{e}_{1}^{\top} V \mathbf{e}_{1}-\lambda_{0}\left(1-\lambda_{0}\right)}{\lambda_{0}^{2}\left(1-\lambda_{0}\right)^{2}}
\end{array}\right)
$$

Recall that $u_{n}=\left(\nabla_{\theta} h\left(\theta_{0}, \lambda_{0}\right), \nabla_{\lambda} h\left(\theta_{0}, \lambda_{0}\right)\right)$. Combining (A.10) and (A.11), we get

$$
\left(\begin{array}{c}
\hat{\theta}-\theta_{0} \\
\hat{\lambda}-\lambda_{0}
\end{array}\right)=\frac{1}{n} W^{-1} u_{n}+o_{p}\left(n^{-1 / 2}\right) .
$$

Note that $|W|=-|V| \cdot\left|\lambda_{0}\left(1-\lambda_{0}\right)\right|=-|V| \cdot\left|\eta_{0}\left(1-\eta_{0}\right)\right|$ and we have assumed that $\eta_{0} \in(0,1)$ and $V$ is nonsingular, the matrix $W$ is nonsingular and its inverse $W^{-1}$ is well defined. Since

$$
W^{-1}=\left(\begin{array}{cc}
V^{-1}-\frac{1}{\lambda_{0}\left(1-\lambda_{0}\right)} \mathbf{e}_{1} \mathbf{e}_{1}^{\top} & \mathbf{e}_{1} \\
\mathbf{e}_{1}^{\top} & -\lambda_{0}\left(1-\lambda_{0}\right)
\end{array}\right)
$$

we have

$$
\hat{\theta}-\theta_{0}=n^{-1}\left(V^{-1}-\frac{1}{\lambda_{0}\left(1-\lambda_{0}\right)} \mathbf{e}_{1} \mathbf{e}_{1}^{\top} \quad \mathbf{e}_{1}\right) u_{n}+o_{p}\left(n^{-1 / 2}\right) .
$$

With Lemma 2, we can verify that

$$
\operatorname{Var}\left\{n^{-1 / 2}\left(V^{-1}-\frac{1}{\lambda_{0}\left(1-\lambda_{0}\right)} \mathbf{e}_{1} \mathbf{e}_{1}^{\top} \quad \mathbf{e}_{1}\right) u_{n}\right\}=V^{-1}-\frac{1}{\lambda_{0}\left(1-\lambda_{0}\right)} \mathbf{e}_{1} \mathbf{e}_{1}^{\top}
$$


Note that $u_{n}$ is the sum of independent and identically distributed random vectors. Hence,

$$
\sqrt{n}\left(\hat{\theta}-\theta_{0}\right) \rightarrow N\left(0, \quad V^{-1}-\frac{1}{\lambda_{0}\left(1-\lambda_{0}\right)} \mathbf{e}_{1} \mathbf{e}_{1}^{\top}\right)
$$

in distribution. This completes the proof of Part (a).

Next, we consider Part (b). Recall that $R(\theta)=2\left\{\ell_{2}(\hat{\theta})-\ell_{2}(\theta)\right\}$. Then $R\left(\theta_{0}\right)=2\{h(\hat{\theta}, \hat{\lambda})-$ $\left.h\left(\theta_{0}, \lambda_{\theta_{0}}\right)\right\}$, where $\lambda_{\theta_{0}}$ is the solution to $\partial h\left(\theta_{0}, \lambda\right) / \partial \lambda=0$.

Applying a second-order Taylor expansion to $h(\hat{\theta}, \hat{\lambda})$ and using (A.12), we have

$$
h(\hat{\theta}, \hat{\lambda})=\frac{n}{2} u_{n}^{\top} W^{-1} u_{n}+o_{p}(1) .
$$

Following a similar argument to that for (A.15), we get

$$
h\left(\theta_{0}, \lambda_{\theta_{0}}\right)=-\frac{n}{2} u_{n 2}^{2} \frac{\lambda_{0}^{2}\left(1-\lambda_{0}\right)^{2}}{\lambda_{0}\left(1-\lambda_{0}\right)-\mathbf{e}_{1}^{\top} V \mathbf{e}_{1}}+o_{p}(1) .
$$

Combining (A.15) and (A.16) gives

$$
R\left(\theta_{0}\right)=n u_{n}^{\top}\left(\begin{array}{cc}
V^{-1}-\frac{\mathbf{e}_{1} \mathbf{e}_{1}^{\top}}{\lambda_{0}\left(1-\lambda_{0}\right)} & \mathbf{e}_{1} \\
\mathbf{e}_{1}^{\top} & \frac{\lambda_{0}\left(1-\lambda_{0}\right) \mathbf{e}_{1}^{\top} V \mathbf{e}_{1}}{\lambda_{0}\left(1-\lambda_{0}\right)-\mathbf{e}_{1}^{\top} V \mathbf{e}_{1}}
\end{array}\right) u_{n}+o_{p}(1) .
$$

Since $W^{-1}$ are invertible, the matrix $V^{-1}-\left\{\lambda_{0}\left(1-\lambda_{0}\right)\right\}^{-1} \mathbf{e}_{1} \mathbf{e}_{1}^{\top}$ is also invertible. Let

$$
v_{n}=u_{n 1}+\left[V^{-1}-\left\{\lambda_{0}\left(1-\lambda_{0}\right)\right\}^{-1} \mathbf{e}_{1} \mathbf{e}_{1}^{\top}\right]^{-1} \mathbf{e}_{1} u_{n 2} .
$$

After some algebra, $R\left(\theta_{0}\right)$ can be written as

$$
R\left(\theta_{0}\right)=n v_{n}^{\top}\left[V^{-1}-\left\{\lambda_{0}\left(1-\lambda_{0}\right)\right\}^{-1} \mathbf{e}_{1} \mathbf{e}_{1}^{\top}\right] v_{n}+o_{p}(1)
$$

With Lemma 2, we can further verify that $\mathbb{E}\left(v_{n}\right)=0$ and

$$
\operatorname{Var}\left(n^{-1 / 2} v_{n}\right)=V+\frac{V \mathbf{e}_{1} \mathbf{e}_{1}^{\top} V}{\lambda_{0}\left(1-\lambda_{0}\right)-\mathbf{e}_{1}^{\top} V \mathbf{e}_{1}}=\left[V^{-1}-\left\{\lambda_{0}\left(1-\lambda_{0}\right)\right\}^{-1} \mathbf{e}_{1} \mathbf{e}_{1}^{\top}\right]^{-1} .
$$

Hence, $R\left(\theta_{0}\right) \rightarrow \chi_{d_{\theta}}^{2}$ in distribution. This completes the proof of Theorem 1 in the main paper. 


\section{Appendix 5: Proof of Theorem 2}

Recall that $\hat{\eta}=n_{1} / n=1-\hat{\lambda}$ with $\eta_{0}=\operatorname{pr}(D=1)$. Then $\hat{\mu}$ in (3.12) can be rewritten as

$$
\begin{aligned}
\hat{\mu} & =\sum_{i=1}^{n} \hat{p}_{i}\left[\int y\left\{\hat{\eta}+(1-\hat{\eta}) \exp \left(\hat{\alpha}+\mathbf{x}_{i}^{\top} \hat{\beta}+\hat{\gamma} y\right)\right\} f\left(y \mid \mathbf{x}_{i}, \hat{\xi}\right) d y\right] \\
& =\frac{1}{n} \sum_{i=1}^{n} \frac{\int y\left\{\hat{\eta}+(1-\hat{\eta}) \exp \left(\hat{\alpha}+\mathbf{x}_{i}^{\top} \hat{\beta}+\hat{\gamma} y\right)\right\} f\left(y \mid \mathbf{x}_{i}, \hat{\xi}\right) d y}{\hat{\eta}+(1-\hat{\eta}) \exp \left\{\hat{\alpha}+\mathbf{x}_{i}^{\top} \hat{\beta}+c\left(\mathbf{x}_{i}, \hat{\gamma}, \hat{\xi}\right)\right\}} \\
& =n^{-1} \sum_{i=1}^{n} K\left(\mathbf{x}_{i} ; \hat{\theta}, \hat{\eta}\right),
\end{aligned}
$$

where

$$
K(\mathbf{x} ; \theta, \eta)=\frac{\int y\left\{\eta+(1-\eta) \exp \left(\alpha+\mathbf{x}^{\top} \beta+\gamma y\right)\right\} f(y \mid \mathbf{x}, \xi) d y}{\eta+(1-\eta) \exp \left\{\alpha+\mathbf{x}^{\top} \beta+c(\mathbf{x}, \gamma, \xi)\right\}} .
$$

Applying the first-order Taylor expansion and the law of large numbers, we have

$$
\hat{\mu}=\frac{1}{n} \sum_{i=1}^{n} K\left(\mathbf{x}_{i} ; \theta_{0}, \eta_{0}\right)+A^{\top}\left(\hat{\theta}-\theta_{0}\right)-B\left\{(1-\hat{\eta})-\left(1-\eta_{0}\right)\right\}+o_{p}\left(n^{-1 / 2}\right),
$$

where $A=\mathbb{E}\left\{\nabla_{\theta} K\left(\mathbf{X} ; \theta_{0}, \eta_{0}\right)\right\}$ and $B=\mathbb{E}\left\{\nabla_{\eta} K\left(\mathbf{X} ; \theta_{0}, \eta_{0}\right)\right\}$. Hence, with Equation (A.12) and $\hat{\eta}=1-\hat{\lambda}$, we have

$$
\hat{\mu}-\mu=\frac{1}{n} \sum_{i=1}^{n}\left\{K\left(\mathbf{x}_{i} ; \theta_{0}, \eta_{0}\right)-\mu\right\}+n^{-1}\left(A^{\top},-B\right) W^{-1} u_{n}+o_{p}\left(n^{-1 / 2}\right) .
$$

We first argue that $E\left\{K\left(\mathbf{X} ; \theta_{0}, \eta_{0}\right)\right\}=\mu$. By (2.6) , we have

$$
\operatorname{pr}(\mathbf{x})=\left\{\eta_{0}+\left(1-\eta_{0}\right) \exp \left\{\alpha_{0}+\mathbf{x}^{\top} \beta_{0}+c\left(\mathbf{x}, \gamma_{0}, \xi_{0}\right)\right\} \operatorname{pr}(\mathbf{x} \mid D=1) .\right.
$$

It then follows that

$$
\begin{aligned}
& \mathbb{E}\left\{K\left(\mathbf{X} ; \theta_{0}, \eta_{0}\right)\right\} \\
= & \int_{\mathbf{X}} \frac{\int_{y} y\left\{\eta_{0}+\left(1-\eta_{0}\right) \exp \left(\alpha_{0}+\mathbf{x}^{\top} \beta_{0}+\gamma_{0} y\right)\right\} f\left(y \mid \mathbf{x}, \xi_{0}\right) d y}{\eta_{0}+\left(1-\eta_{0}\right) \exp \left\{\alpha_{0}+\mathbf{x}^{\top} \beta_{0}+c\left(\mathbf{x}, \gamma_{0}, \xi_{0}\right)\right\}} \operatorname{pr}(\mathbf{x}) d \mathbf{x} \\
= & \int_{\mathbf{X}} \int_{y} y\left\{\eta_{0}+\left(1-\eta_{0}\right) \exp \left(\alpha_{0}+\mathbf{x}^{\top} \beta_{0}+\gamma_{0} y\right)\right\} f\left(y \mid \mathbf{x}, \xi_{0}\right) \operatorname{pr}(\mathbf{x} \mid D=1) d y d \mathbf{x} \\
= & \int_{\mathbf{X}} \int_{y} y\left\{\eta_{0}+\left(1-\eta_{0}\right) \exp \left(\alpha_{0}+\mathbf{x}^{\top} \beta_{0}+\gamma_{0} y\right)\right\} \operatorname{pr}(y, \mathbf{x} \mid D=1) d y d \mathbf{x} \\
= & \int_{\mathbf{X}, y} y \operatorname{pr}(y, \mathbf{x}) d y d \mathbf{x}=\mu .
\end{aligned}
$$


After some calculus, we found that $-B=A^{\top} \mathbf{e}_{1} /\left\{\left(1-\eta_{0}\right) \eta_{0}\right\}$. With (A.13), we have

$$
\begin{aligned}
& \left(A^{\top},-B\right) W^{-1} u_{n} \\
= & A^{\top}\left(I, \frac{\mathbf{e}_{1}}{\lambda_{0}\left(1-\lambda_{0}\right)}\right)\left(\begin{array}{cc}
V^{-1}-\frac{1}{\lambda_{0}\left(1-\lambda_{0}\right)} \mathbf{e}_{1} \mathbf{e}_{1}^{\top} & \mathbf{e}_{1} \\
\mathbf{e}_{1}^{\top} & -\lambda_{0}\left(1-\lambda_{0}\right)
\end{array}\right)\left(\begin{array}{l}
u_{n 1} \\
u_{n 2}
\end{array}\right) \\
= & A^{\top} V^{-1} u_{n 1} .
\end{aligned}
$$

Since $\mathbb{E}\left(u_{n 1} \mid \mathbf{x}_{1}, \ldots, \mathbf{x}_{n}\right)=0$, we arrive at

$$
\begin{aligned}
& \operatorname{Cov}\left(\sum_{i=1}^{n} K\left(\mathbf{x}_{i} ; \theta_{0}, \eta_{0}\right),\left(A^{\top},-B\right) W^{-1} u_{n}\right) \\
= & \operatorname{Cov}\left(\sum_{i=1}^{n} K\left(\mathbf{x}_{i} ; \theta_{0}, \eta_{0}\right), A^{\top} V^{-1} u_{n 1}\right) \\
= & 0 .
\end{aligned}
$$

Finally, By Lemma 2 and the central limit theorem and Slutsky's theorem, we have

$$
\sqrt{n}(\hat{\mu}-\mu) \rightarrow N\left(0, \sigma^{2}\right)
$$

where $\sigma^{2}=\operatorname{Var}\left\{K\left(\mathbf{X} ; \theta_{0}, \eta_{0}\right)\right\}+A^{\top} V^{-1} A$. This proves Theorem 2 of the main paper.

\section{Appendix 6: Proof of Theorem 3}

\section{Preparations}

The observed data are $\left(d_{i}=1, \mathbf{x}_{i}, y_{i}\right)\left(i=1, \ldots, n_{1}\right)$ and $\left(d_{i}=0, \mathbf{x}_{i}\right)\left(i=n_{1}+1, \ldots, n\right)$. We make two parametric assumptions:

$$
\begin{aligned}
& \operatorname{pr}(D=1 \mid \mathbf{X}=\mathbf{x}, Y=y)=\frac{1}{1+\exp \left\{\alpha^{*}+\mathbf{x}^{\top} \beta+\gamma y\right\}} \\
& \operatorname{pr}(Y=y \mid \mathbf{X}=\mathbf{x}, D=1)=f(y \mid \mathbf{x}, \xi)
\end{aligned}
$$

Recall that $\eta=\operatorname{pr}(D=1)$ and $c(\mathbf{x}, \gamma, \xi)=\log \int e^{\gamma y} f(y \mid \mathbf{x}, \xi) d y$. Let $\vartheta=(\beta, \gamma, \xi)$ and $r(\mathbf{x}, \vartheta)=\mathbf{x}^{\top} \beta+c(\mathbf{x}, \gamma, \xi)$, so that $\theta=\left(\alpha, \vartheta^{\top}\right)^{\top}$ and $t(\mathbf{x}, \theta)=\alpha+r(\mathbf{x}, \vartheta)$. We have shown

$$
\begin{aligned}
\operatorname{pr}(y, \mathbf{x} \mid D=0) & =\exp \left\{\alpha+\mathbf{x}^{\top} \beta+\gamma y\right\} \operatorname{pr}(y, \mathbf{x} \mid D=1) \\
\operatorname{pr}(\mathbf{X}=\mathbf{x} \mid D=0) & =\exp \{\alpha+r(\mathbf{x}, \vartheta)\} \operatorname{pr}(\mathbf{X}=\mathbf{x} \mid D=1)
\end{aligned}
$$


where $\alpha=\alpha^{*}+\log \{\eta /(1-\eta)\}$.

In addition,

$$
\mathbb{E}(1-D \mid \mathbf{X}=\mathbf{x})=\operatorname{pr}(D=0 \mid \mathbf{X}=\mathbf{x})=\pi(\mathbf{x} ; \alpha, \vartheta, \eta)
$$

where we have defined

$$
\pi(\mathbf{x} ; \alpha, \vartheta, \eta)=\frac{(1-\eta) \exp \{t(\mathbf{x}, \theta)\}}{\eta+(1-\eta) \exp \{t(\mathbf{x}, \theta)\}}=\frac{(1-\eta) \exp \{\alpha+r(\mathbf{x}, \vartheta)\}}{\eta+(1-\eta) \exp \{\alpha+r(\mathbf{x}, \vartheta)\}}
$$

with $\pi(\mathbf{x})$ abbreviation for $\pi\left(\mathbf{x} ; \theta_{0}, \eta_{0}\right)$.

The observed data are iid from $(D, \mathbf{X}, \tilde{Y})$, where $\tilde{Y}$ is empty when $D=0$, and $\tilde{Y}=Y$ when $D=1$. The joint distribution of $(D, \mathbf{X}, \tilde{Y})$ is

$$
\begin{aligned}
& \{\operatorname{pr}(Y=y \mid \mathbf{X}=\mathbf{x}, D=1) \operatorname{pr}(\mathbf{X}=\mathbf{x} \mid D=1) \operatorname{pr}(D=1)\}^{d} \\
& \times\{\operatorname{pr}(\mathbf{X}=\mathbf{x} \mid D=0) \operatorname{pr}(D=0)\}^{1-d} \\
= & \{\operatorname{pr}(Y=y \mid \mathbf{X}=\mathbf{x}, D=1) \operatorname{pr}(D=1)\}^{d} \\
& \times\{\exp (t(\mathbf{x}, \theta)) \operatorname{pr}(D=0)\}^{1-d} \times \operatorname{pr}(\mathbf{x} \mid D=1) \\
= & \{f(y \mid \mathbf{x}, \xi) \eta\}^{d} \times\{\exp (\alpha+r(\mathbf{x}, \vartheta))(1-\eta)\}^{1-d} \times \operatorname{pr}(\mathbf{x} \mid D=1) .
\end{aligned}
$$

Here all except $\operatorname{pr}(\mathbf{X}=\mathbf{x} \mid D=1)$ are completely parametric, and we regard $\operatorname{pr}(\mathbf{X}=\mathbf{x} \mid D=1)$ as an infinite-dimensional parameter, or simply

$$
\operatorname{pr}(\mathbf{X}=\mathbf{x} \mid D=1) \geq 0, \quad \int \operatorname{pr}(\mathbf{X}=\mathbf{x} \mid D=1) d \mathbf{x}=1
$$

Therefore our imposed model is clearly semi-parametric.

Throughout this section, we use $g(\mathbf{x}, \zeta)$ to denote a parametric submodel for $\operatorname{pr}(\mathbf{X}=$ $\mathbf{x} \mid D=1)$ with $g\left(\mathbf{x}, \zeta_{0}\right)$ being the true model. The joint density function of $(D, \mathbf{X}, \tilde{Y})$ is

$$
h(d, \mathbf{x}, y ; \alpha, \vartheta, \eta, \zeta)=\{f(y \mid \mathbf{x}, \xi) \eta\}^{d} \times\{\exp (\alpha+r(\mathbf{x}, \vartheta))(1-\eta)\}^{1-d} \times g(\mathbf{x}, \zeta) .
$$

It is worth noting that $\alpha$ is not free but is a function of $(\vartheta, \zeta)$ and determined by

$$
1=\int \exp \{\alpha+r(\mathbf{x}, \vartheta)\} g(\mathbf{x}, \zeta) d \mathbf{x}
$$


The following three functions will be useful in our proof:

$$
\begin{aligned}
B_{1}(d, \mathbf{x}, y) & =\frac{\partial \log h\left(d, \mathbf{x}, y ; \alpha_{0}, \vartheta_{0}, \eta_{0}, \zeta_{0}\right)}{\partial \vartheta} \\
& =(1-d)\left\{\nabla_{\vartheta} \alpha\left(\vartheta_{0}\right)+\nabla_{\vartheta} r\left(\mathbf{x}, \vartheta_{0}\right)\right\}+d I_{e,-1} \nabla_{\xi} \log f\left(y \mid \mathbf{x}, \xi_{0}\right), \\
B_{2}(d, \mathbf{x}, y) & =\frac{\partial \log h\left(d, \mathbf{x}, y ; \alpha_{0}, \vartheta_{0}, \eta_{0}, \zeta_{0}\right)}{\partial \eta}=\frac{D-\eta_{0}}{\eta_{0}\left(1-\eta_{0}\right)} \\
B_{3}(d, \mathbf{x}, y) & =\frac{\partial \log h\left(d, \mathbf{x}, y ; \alpha_{0}, \vartheta_{0}, \eta_{0}, \zeta_{0}\right)}{\partial \zeta}=\nabla_{\zeta} \log g\left(\mathbf{x}, \zeta_{0}\right),
\end{aligned}
$$

where $I_{e,-1}$ is $I_{e}$ without the first row.

\section{Semiparametric efficiency of $(\hat{\theta}, \hat{\eta})$}

We have shown that

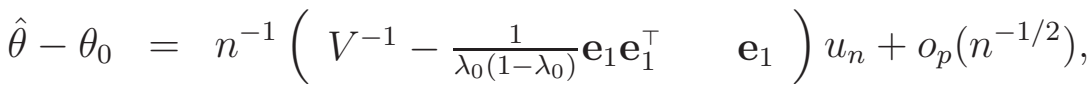

$$
\begin{aligned}
& \hat{\eta}-\eta_{0}=\frac{1}{n} \sum_{i=1}^{n}\left(d_{i}-\eta_{0}\right)
\end{aligned}
$$

where $u_{n}=\left(u_{n 1}^{\top}, u_{n 2}\right)^{\top}$ with

$$
\begin{aligned}
& u_{n 1}=\sum_{i=1}^{n}\left[\left(1-d_{i}-\pi_{i}\right) \nabla_{\theta} t\left(\mathbf{x}_{i}, \theta_{0}\right)+d_{i} I_{e} \nabla_{\xi} \log \left\{f\left(y_{i} \mid \mathbf{x}_{i}, \xi_{0}\right)\right\}\right], \\
& u_{n 2}=\frac{1}{\lambda_{0}\left(1-\lambda_{0}\right)} \sum_{i=1}^{n}\left(\lambda_{0}-\pi_{i}\right) .
\end{aligned}
$$

Therefore

$$
\begin{aligned}
\hat{\theta}-\theta_{0}= & n^{-1}\left\{V^{-1} u_{n 1}-\frac{1}{\lambda_{0}\left(1-\lambda_{0}\right)} \mathbf{e}_{1} \mathbf{e}_{1}^{\top} u_{n 1}+\mathbf{e}_{1} u_{n 2}\right\}+o_{p}\left(n^{-1 / 2}\right) \\
= & n^{-1}\left\{V^{-1} u_{n 1}+\frac{1}{\eta_{0}\left(1-\eta_{0}\right)} \mathbf{e}_{1} \sum_{i=1}^{n}\left(d_{i}-\eta_{0}\right)\right\}+o_{p}\left(n^{-1 / 2}\right) \\
= & n^{-1} \sum_{i=1}^{n}\left[V^{-1}\left(1-d_{i}-\pi_{i}\right) \nabla_{\theta} t\left(\mathbf{x}_{i}, \theta_{0}\right)+V^{-1} d_{i} I_{e} \nabla_{\xi} \log \left\{f\left(y_{i} \mid \mathbf{x}_{i}, \xi_{0}\right)\right\}\right. \\
& \left.\left.+\frac{1}{\eta_{0}\left(1-\eta_{0}\right)} \mathbf{e}_{1}\left(d_{i}-\eta_{0}\right)\right\}\right]+o_{p}\left(n^{-1 / 2}\right) .
\end{aligned}
$$

Then the respective influence functions of $\hat{\theta}$ and $\hat{\eta}$ are

$$
\begin{aligned}
\varphi_{\theta}(D, \mathbf{X}, Y)= & V^{-1}(1-D-\pi(\mathbf{X})) \nabla_{\theta} t\left(\mathbf{X}, \theta_{0}\right)+V^{-1} D I_{e} \nabla_{\xi} \log \left\{f\left(Y \mid \mathbf{X}, \xi_{0}\right)\right\} \\
& +\frac{\left(D-\eta_{0}\right)}{\eta_{0}\left(1-\eta_{0}\right)} \mathbf{e}_{1}
\end{aligned}
$$


and $\varphi_{\eta}(D, \mathbf{X}, Y)=D-\eta_{0}$. We prove only the semiparametric efficiency of $\hat{\theta}$; the semiparametric efficiency of $\hat{\eta}$ can be proved in the same way with less algebra.

Referring to the established theory for the semiparametric efficiency bound, for example Chapter 3 of Bickel et al (1992) and Newey (1990), we need to show only the following two results to establish the semiparametric efficiency of $\hat{\theta}$ :

(a) $\hat{\theta}$ is a regular estimator of $\theta_{0}$;

(b) there exists a parametric submodel with $h_{\psi}(d, \mathbf{x}, \tilde{y})$ the joint density of $(D, \mathbf{X}, \tilde{Y})$ such that the true model is $h_{0}(d, \mathbf{x}, \tilde{y})$ and

$$
\varphi_{\theta}(d, \mathbf{x}, y)=\left.\frac{\partial \log h_{\psi}(d, \mathbf{x}, \tilde{y})}{\partial \psi}\right|_{\psi=0} .
$$

\section{Proof of (a)}

By Theorem 2 in Newey (1990), arguing $\hat{\theta}$ is a regular estimator of $\theta_{0}$ is equivalent to showing that

$$
\begin{aligned}
Z_{1} & \equiv \mathbb{E}\left\{\varphi_{\theta}(D, \mathbf{X}, Y) B_{1}^{\top}(D, \mathbf{X}, Y)\right\} \\
& =\left.\frac{\partial \theta}{\partial \vartheta^{\top}}\right|_{\left(\theta_{0}, \eta_{0}, \zeta_{0}\right)}=\left(\nabla_{\vartheta} \alpha, I_{d_{\vartheta}}\right)^{\top}, \\
Z_{2} & \equiv \mathbb{E}\left\{\varphi_{\theta}(D, \mathbf{X}, Y) B_{2}(D, \mathbf{X}, Y)\right\}=\left.\frac{\partial \theta}{\partial \eta}\right|_{\left(\theta_{0}, \eta_{0}, \zeta_{0}\right)}=0, \\
Z_{3} & \equiv \mathbb{E}\left\{\varphi_{\theta}(D, \mathbf{X}, Y) B_{3}^{\top}(D, \mathbf{X}, Y)\right\}=\left.\frac{\partial \theta}{\partial \zeta^{\top}}\right|_{\left(\theta_{0}, \eta_{0}, \zeta_{0}\right)}=0,
\end{aligned}
$$

where throughout this section $\mathbb{E}$ takes expectation with respect to $h\left(d, \mathbf{x}, y ; \theta_{0}, \eta_{0}, \zeta_{0}\right)$.

(1) Proof of Equality (A.19)

Since $\mathbb{E}\left\{D \nabla_{\xi} \log f\left(Y \mid \mathbf{X}, \xi_{0}\right) \mid \mathbf{X}\right\}=0$, it follows that

$$
\begin{aligned}
Z_{1}= & \mathbb{E}\left\{\varphi_{\theta}(D, \mathbf{X}, Y) B_{1}^{\top}(D, \mathbf{X}, Y)\right\} \\
= & \mathbb{E}\left[(1-D-\pi(\mathbf{X})) V^{-1} \nabla_{\theta} t\left(\mathbf{X}, \theta_{0}\right)(1-D)\left\{\nabla_{\vartheta} \alpha\left(\vartheta_{0}\right)+\nabla_{\vartheta} r\left(\mathbf{X}, \theta_{0}\right)\right\}^{\top}\right] \\
& +\mathbb{E}\left[(1-D) r\left(\mathbf{X}, \theta_{0}\right) \frac{\left(D-\eta_{0}\right)}{\eta_{0}\left(1-\eta_{0}\right)} \mathbf{e}_{1}\left\{\nabla_{\vartheta} \alpha\left(\vartheta_{0}\right)+\nabla_{\vartheta}\right\}^{\top}\right] \\
& +\mathbb{E}\left[D V^{-1} I_{e} \nabla_{\xi}\left\{\log f\left(Y \mid \mathbf{X}, \xi_{0}\right)\right\}^{\otimes 2} I_{e,-1}^{\top}\right]
\end{aligned}
$$




$$
\begin{aligned}
= & \mathbb{E}\left[\pi(\mathbf{X})(1-\pi(\mathbf{X})) V^{-1} \nabla_{\theta} t\left(\mathbf{X}, \theta_{0}\right)\left\{\nabla_{\vartheta} \alpha\left(\vartheta_{0}\right)+\nabla_{\vartheta} r\left(\mathbf{X}, \theta_{0}\right)\right\}^{\top}\right] \\
& \left.-\mathbb{E}\left[\frac{(1-D) \eta_{0}}{\eta_{0}\left(1-\eta_{0}\right)} \mathbf{e}_{1}\left\{\nabla_{\vartheta} \alpha\left(\vartheta_{0}\right)+\nabla_{\vartheta} r\left(\mathbf{X}, \theta_{0}\right)\right\}^{\top}\right\}\right] \\
& +\mathbb{E}\left[D I_{e} \nabla_{\xi}\left\{\log f\left(Y \mid \mathbf{X}, \xi_{0}\right)\right\}^{\otimes 2} I_{e,-1}^{\top} V^{-1}\right] \\
= & \left.\mathbf{e}_{1}\left\{\nabla_{\vartheta} \alpha\left(\vartheta_{0}\right)\right\}^{\top}+\mathbb{E}\left[\frac{\pi(\mathbf{X})}{1-\eta_{0}} \mathbf{e}_{1}\left\{\nabla_{\vartheta} \alpha\left(\vartheta_{0}\right)+\nabla_{\vartheta} r\left(\mathbf{X}, \theta_{0}\right)\right\}^{\top}\right\}\right]+I_{e} I_{e,-1}^{\top},
\end{aligned}
$$

where we have used the definition

$$
V=\mathbb{E}\left[\{1-\pi(\mathbf{X})\} \pi(\mathbf{X})\left\{\nabla_{\theta} t(\mathbf{X}, \theta)\right\}^{\otimes 2}\right]+\mathbb{E}\left[D I_{e}\left\{\nabla_{\xi} f(Y \mid \mathbf{X}, \xi)\right\}^{\otimes 2} I_{e}^{\top}\right] .
$$

Taking derivative with respect to $\vartheta$ on both sides of (A.18) gives

$$
0=\int\left\{\nabla_{\vartheta} \alpha\left(\vartheta_{0}\right)+\nabla_{\vartheta} r\left(\mathbf{x}, \vartheta_{0}\right)\right\} \exp \left\{\alpha\left(\vartheta_{0}\right)+r\left(\mathbf{x}, \vartheta_{0}\right)\right\} g\left(\mathbf{x}, \zeta_{0}\right) d \mathbf{x} .
$$

This together with $g\left(\mathbf{x}, \zeta_{0}\right) d \mathbf{x}=d F(\mathbf{x} \mid D=1)$ leads to

$$
\begin{aligned}
& \left.\frac{1}{1-\eta_{0}} \mathbf{e}_{1}^{\top} \mathbb{E}\left[\left\{\nabla_{\vartheta} \alpha\left(\vartheta_{0}\right)+\nabla_{\vartheta} r\left(\mathbf{X}, \theta_{0}\right)\right\} \pi(\mathbf{X})\right\}\right] \\
= & \frac{\eta_{0}}{1-\eta_{0}} \mathbf{e}_{1}^{\top} \int\left\{\nabla_{\vartheta} \alpha\left(\vartheta_{0}\right)+\nabla_{\vartheta} r\left(\mathbf{x}, \vartheta_{0}\right)\right\} \exp \left\{\alpha\left(\vartheta_{0}\right)+r\left(\mathbf{x}, \vartheta_{0}\right)\right\} g\left(\mathbf{x}, \zeta_{0}\right) d \mathbf{x} \\
= & 0 .
\end{aligned}
$$

Therefore, we have

$$
Z_{1}=\nabla_{\vartheta} \alpha\left(\vartheta_{0}\right) e_{1}^{\top}+I_{e} I_{e,-1}^{\top}=\left(\nabla_{\vartheta} \alpha, I_{d_{\vartheta}}^{\top}\right)^{\top}
$$

This proves (A.19).

(2) Proof of Equality (A.20)

Since $\mathbb{E} \varphi_{\theta}(D, \mathbf{X}, Y)=0$, we have

$$
\begin{aligned}
Z_{2}= & \mathbb{E}\left\{B_{2}(D, \mathbf{X}, Y) \varphi_{\theta}(D, \mathbf{X}, Y)\right\} \\
= & \frac{1}{\eta_{0}\left(1-\eta_{0}\right)} \mathbb{E}\left\{D \varphi_{\theta}(D, \mathbf{X}, Y)\right\} \\
= & \frac{1}{\eta_{0}\left(1-\eta_{0}\right)} \mathbb{E}\left\{-D V^{-1} \pi(\mathbf{X}) \nabla_{\theta} t\left(\mathbf{X}, \theta_{0}\right)+D \frac{1}{\eta_{0}\left(1-\eta_{0}\right)} \mathbf{e}_{1}\left(1-\eta_{0}\right)\right\} \\
& \left.+D V^{-1} I_{e} \nabla_{\xi} \log \left\{f\left(Y \mid \mathbf{X}, \xi_{0}\right)\right\}\right\} \\
= & \frac{1}{\eta_{0}\left(1-\eta_{0}\right)} \mathbb{E}\left\{-V^{-1}(1-\pi(\mathbf{X})) \pi(\mathbf{X}) \nabla_{\theta} t\left(\mathbf{X}, \theta_{0}\right)+\mathbf{e}_{1}\right\} \\
= & 0,
\end{aligned}
$$


where the last equality holds because

$$
\mathbb{E}\left\{\pi(\mathbf{X})(1-\pi(\mathbf{X})) \nabla_{\theta} t\left(\mathbf{X}, \theta_{0}\right)\right\}=V e_{1}
$$

This proves Equality (A.20).

(3) Proof of Equality (A.21)

Since

$$
\mathbb{E}\left\{\varphi_{\theta}(D, \mathbf{X}, Y) \mid \mathbf{x}\right\}=\frac{1}{\eta_{0}\left(1-\eta_{0}\right)} \mathbf{e}_{1}\left\{1-\eta_{0}-\pi(\mathbf{X})\right\}
$$

we have

$$
\begin{aligned}
Z_{3} & =\mathbb{E}\left\{\varphi_{\theta}(D, \mathbf{X}, Y) B_{3}^{\top}(D, \mathbf{X}, Y)\right\} \\
& =\mathbb{E}\left[\frac{1}{\eta_{0}\left(1-\eta_{0}\right)} \mathbf{e}_{1}\left\{1-\eta_{0}-\pi(\mathbf{X})\right\} \nabla_{\zeta^{\top}} \log g\left(\mathbf{X}, \zeta_{0}\right)\right] \\
& =-\frac{1}{\eta_{0}\left(1-\eta_{0}\right)} \mathbb{E}\left[\mathbf{e}_{1} \pi(\mathbf{X}) \nabla_{\zeta^{\top}} \log g\left(\mathbf{X}, \zeta_{0}\right)\right]
\end{aligned}
$$

Taking derivative with respect to $\zeta$ on both sides of Eq (A.18) gives

$$
\begin{aligned}
0 & =\int \exp \left\{\alpha_{0}+r\left(\mathbf{x}, \vartheta_{0}\right)\right\}\left\{\nabla_{\zeta} \log g\left(\mathbf{x}, \zeta_{0}\right)\right\} g\left(\mathbf{x}, \zeta_{0}\right) d \mathbf{x} \\
& =\int \exp \left\{\alpha_{0}+r\left(\mathbf{x}, \vartheta_{0}\right)\right\}\left\{\nabla_{\zeta} \log g\left(\mathbf{x}, \zeta_{0}\right)\right\} \frac{1-\pi(\mathbf{x})}{\eta_{0}} \operatorname{pr}(\mathbf{x}) d \mathbf{x} \\
& =\int\left\{\nabla_{\zeta} \log g\left(\mathbf{x}, \zeta_{0}\right)\right\} \frac{\pi(\mathbf{x})}{\eta_{0}\left(1-\eta_{0}\right)} \operatorname{pr}(\mathbf{x}) d \mathbf{x} \\
& =\mathbb{E}\left[\left\{\nabla_{\zeta} \log g\left(\mathbf{X}, \zeta_{0}\right)\right\} \frac{\pi(\mathbf{X})}{\eta_{0}\left(1-\eta_{0}\right)}\right],
\end{aligned}
$$

which means $Z_{3}=0$. This proves Equality (A.21) and also completes the proof of (a).

\section{Proof of (b)}

Consider the following function

$$
\begin{aligned}
h_{\psi}(d, \mathbf{x}, \tilde{y})= & \left\{1+\psi \varphi_{\theta}(d, \mathbf{x}, y)\right\} \times\left\{f\left(y \mid \mathbf{x}, \xi_{0}\right) \eta_{0}\right\}^{d} \\
& \times\left\{\exp \left(\alpha_{0}+r\left(\mathbf{x}, \vartheta_{0}\right)\right)\left(1-\eta_{0}\right)\right\}^{1-d} g\left(\mathbf{x}, \zeta_{0}\right) .
\end{aligned}
$$

Suppose the support of $(\mathbf{X}, Y)$ is compact, then it can be verified that the function

$$
\begin{aligned}
\varphi_{\theta}(D, \mathbf{X}, Y)= & V^{-1}(1-D-\pi(\mathbf{X})) \nabla_{\theta} t\left(\mathbf{X}, \theta_{0}\right)+\frac{1}{\eta_{0}\left(1-\eta_{0}\right)} \mathbf{e}_{1}\left(D-\eta_{0}\right) \\
& +V^{-1} D I_{e} \nabla_{\xi} \log \left\{f\left(Y \mid \mathbf{X}, \xi_{0}\right)\right\}
\end{aligned}
$$


is bounded. Because $\mathbb{E}\left\{\varphi_{\theta}(D, \mathbf{X}, Y)\right\}=0$ where $\mathbb{E}$ takes expectation with respect to $h\left(d, \mathbf{x}, y ; \alpha_{0}, \vartheta_{0}, \eta_{0}, \zeta_{0}\right)$, the function $h_{\psi}(d, \mathbf{x}, \tilde{y})$ is a density function when $\psi$ is small enough. When $\psi=0$, it reduces to the true joint density function $h\left(d, \mathbf{x}, y ; \alpha_{0}, \vartheta_{0}, \eta_{0}, \zeta_{0}\right)$. It is easy to check that $h_{\psi}(d, \mathbf{x}, \tilde{y})$ with small enough $\psi$ is a parametric submodel and

$$
\left.\nabla_{\psi} h_{\psi}(d, \mathbf{x}, \tilde{y})\right|_{\psi=0}=\varphi_{\theta}(d, \mathbf{x}, y)
$$

This proves (b), and hence proves the semiparametric efficiency of $\hat{\theta}$.

\section{Semiparametric efficiency of $\hat{\mu}$}

The population mean can be expressed as

$$
\begin{aligned}
\mu= & \int_{y} \int_{\mathbf{X}} y \operatorname{pr}(y \mid \mathbf{x}, D=1) \operatorname{pr}(\mathbf{x} \mid D=1) \operatorname{pr}(D=1) d \mathbf{x} d y \\
& +\int_{y} \int_{\mathbf{X}} y \operatorname{pr}(y \mid \mathbf{x}, D=0) \operatorname{pr}(\mathbf{x} \mid D=0) \operatorname{pr}(D=0) d \mathbf{x} d y \\
= & \int_{y} \int_{\mathbf{X}} y \operatorname{pr}(y \mid \mathbf{x}, D=1) \operatorname{pr}(\mathbf{x} \mid D=1) \eta d \mathbf{x} d y \\
& +\int_{y} \int_{\mathbf{X}} y \exp \left(\alpha+\mathbf{x}^{\top} \beta+\gamma y\right) \operatorname{pr}(y \mid \mathbf{x}, D=1) \operatorname{pr}(\mathbf{x} \mid D=1)(1-\eta) d \mathbf{x} d y \\
= & \int_{\mathbf{X}}\left[\int_{y} y\left\{\eta+(1-\eta) \exp \left(\alpha+\mathbf{x}^{\top} \beta+\gamma y\right)\right\} f(y \mid \mathbf{x}, \xi) d y\right] d F(\mathbf{x} \mid D=1)
\end{aligned}
$$

The proposed mean estimator is

$$
\begin{aligned}
\hat{\mu} & =\frac{1}{n} \sum_{i=1}^{n} \frac{\int_{y} y\left\{\hat{\eta}+(1-\hat{\eta}) \exp \left(\hat{\alpha}+\mathbf{x}_{i}^{\top} \hat{\beta}+\hat{\gamma} y\right)\right\} f\left(y \mid \mathbf{x}_{i}, \hat{\xi}\right) d y}{\hat{\eta}+(1-\hat{\eta}) \exp \left\{\hat{\alpha}+\mathbf{x}_{i}^{\top} \hat{\beta}+c\left(\mathbf{x}_{i}, \hat{\gamma}, \hat{\xi}\right)\right\}} \\
& =n^{-1} \sum_{i=1}^{n} K\left(\mathbf{x}_{i} ; \hat{\theta}, \hat{\eta}\right),
\end{aligned}
$$

where

$$
K(\mathbf{x} ; \theta, \eta)=\frac{\int y\left\{\eta+(1-\eta) \exp \left(\alpha+\mathbf{x}^{\top} \beta+\gamma y\right)\right\} f(y \mid \mathbf{x}, \xi) d y}{\eta+(1-\eta) \exp \left\{\alpha+\mathbf{x}^{\top} \beta+c(\mathbf{x}, \gamma, \xi)\right\}} .
$$

Recall that $A=\mathbb{E}\left\{\nabla_{\theta} K\left(\mathbf{X} ; \theta_{0}, \eta_{0}\right)\right\}, \pi_{i}=\pi\left(\mathbf{x}_{i}\right)$ and $\left.I_{e}^{\top}=\left(0_{d_{\xi} \times\left(2+d_{\beta}\right.}\right), I_{d_{\xi} \times d_{\xi}}\right)$. We have shown in the proof of Theorem 2 that

$$
\hat{\mu}-\mu=\frac{1}{n} \sum_{i=1}^{n}\left\{K\left(\mathbf{x}_{i} ; \theta_{0}, \eta_{0}\right)-\mu\right\}+n^{-1} A^{\top} V^{-1} u_{n 1}+o_{p}\left(n^{-1 / 2}\right),
$$


where $u_{n 1}=\sum_{i=1}^{n}\left[\left(1-d_{i}-\pi_{i}\right) \nabla_{\theta} t\left(\mathbf{x}_{i}, \theta_{0}\right)+d_{i} I_{e} \nabla_{\xi} \log \left\{f\left(y_{i} \mid \mathbf{x}_{i}, \xi_{0}\right)\right\}\right]$. Equivalently

$$
\begin{aligned}
\hat{\mu}-\mu= & \frac{1}{n} \sum_{i=1}^{n}\left\{K\left(\mathbf{x}_{i} ; \theta_{0}, \eta_{0}\right)-\mu_{0}+\left(1-d_{i}-\pi_{i}\right) A^{\top} V^{-1} \nabla_{\theta} t\left(\mathbf{x}_{i}, \theta_{0}\right)\right. \\
& \left.+d_{i} A^{\top} V^{-1} I_{e} \nabla_{\xi} \log f\left(y_{i} \mid \mathbf{x}_{i}, \xi_{0}\right)\right\}+o_{p}\left(n^{-1 / 2}\right),
\end{aligned}
$$

which implies that the influence function of $\hat{\mu}$ is

$$
\begin{aligned}
\varphi_{\mu}(D, \mathbf{X}, Y)= & K\left(\mathbf{X} ; \theta_{0}, \eta_{0}\right)-\mu_{0}+\{1-D-\pi(\mathbf{X})\} A^{\top} V^{-1} \nabla_{\theta} t\left(\mathbf{X}, \theta_{0}\right) \\
& +D A^{\top} V^{-1} I_{e} \nabla_{\xi} \log f\left(Y \mid \mathbf{X}, \xi_{0}\right) .
\end{aligned}
$$

Similar to the proof of the semiparametric efficiency of $\hat{\theta}$, we need to show only the following two results to establish the semiparametric efficiency of $\hat{\mu}$ :

(a1) $\hat{\mu}$ is a regular estimator of $\mu_{0}$;

(b1) there exists a parametric submodel with $h_{\psi}^{*}(d, \mathbf{x}, \tilde{y})$ the joint density of $(D, \mathbf{X}, \tilde{Y})$ such that the true model is $h_{0}^{*}(d, \mathbf{x}, \tilde{y})$ and

$$
\varphi_{\mu}(d, \mathbf{x}, y)=\left.\frac{\partial \log h_{\psi}^{*}(d, \mathbf{x}, \tilde{y})}{\partial \psi}\right|_{\psi=0} .
$$

\section{Proof of (a1)}

Under the submodel $g(\mathbf{x}, \zeta)$ for $\operatorname{pr}(\mathbf{X}=\mathbf{x} \mid D=1)$, we can write $\mu$ as

$$
\mu=\mu(\theta, \eta, \zeta) \equiv \int_{\mathbf{X}}\left[\int_{y} y\left\{\eta+(1-\eta) \exp \left(\alpha+\mathbf{x}^{\top} \beta+\gamma y\right)\right\} f(y \mid \mathbf{x}, \xi) d y\right] g(\mathbf{x}, \zeta) d \mathbf{x} .
$$

Define $w(\mathbf{x}, y)=\left(\mathbf{x}^{\top}, y, \nabla_{\xi^{\top}} \log f\left(y \mid \mathbf{x}, \xi_{0}\right)\right)^{\top}$. The partial derivative of $\mu$ is

$$
\begin{aligned}
\nabla_{\vartheta} \mu\left(\theta_{0}, \eta_{0}\right)= & \int_{\mathbf{x}} \int_{y} y\left\{\eta_{0}+\left(1-\eta_{0}\right) \exp \left(\alpha_{0}+\mathbf{x}^{\top} \beta_{0}+\gamma_{0} y\right)\right\} \\
& \times\left\{\nabla_{\vartheta} \alpha\left(\vartheta_{0}\right)+w(\mathbf{x}, y)\right\} f\left(y \mid \mathbf{x}, \xi_{0}\right) d y g(\mathbf{x}, \zeta) d \mathbf{x} .
\end{aligned}
$$

By Theorem 2 in Newey (1990), arguing $\hat{\mu}$ is a regular estimator of $\mu_{0}$ is equivalent to showing that

$$
\begin{aligned}
C_{1} & \equiv \mathbb{E}\left\{\varphi_{\mu}(D, \mathbf{X}, Y) B_{1}(D, \mathbf{X}, Y)\right\}=\frac{\partial \mu\left(\theta_{0}, \eta_{0}, \zeta_{0}\right)}{\partial \vartheta}, \\
C_{2} & \equiv \mathbb{E}\left\{\varphi_{\mu}(D, \mathbf{X}, Y) B_{2}(D, \mathbf{X}, Y)\right\}=\frac{\partial \mu\left(\theta_{0}, \eta_{0}, \zeta_{0}\right)}{\partial \eta}, \\
C_{3} & \equiv \mathbb{E}\left\{\varphi_{\mu}(D, \mathbf{X}, Y) B_{3}(D, \mathbf{X}, Y)\right\}=\frac{\partial \mu\left(\theta_{0}, \eta_{0}, \zeta_{0}\right)}{\partial \zeta},
\end{aligned}
$$


where $\mathbb{E}$ takes expectation with respect to $h\left(d, \mathbf{x}, y ; \theta_{0}, \eta_{0}, \zeta_{0}\right)$. Keep in mind that $\alpha$ is a function of $\vartheta$ and $\zeta$.

(1) Proof of Equality (A.22)

Since $\mathbb{E}\left\{D \nabla_{\xi} \log f\left(Y \mid \mathbf{X}, \xi_{0}\right) \mid \mathbf{X}\right\}=0$, it follows that

$$
\begin{aligned}
C_{1}= & \mathbb{E}\left\{\varphi_{\mu}(D, \mathbf{X}, Y) B_{1}(D, \mathbf{X}, Y)\right\} \\
= & \mathbb{E}\left[(1-D) \nabla_{\theta}\left\{\nabla_{\vartheta} \alpha\left(\vartheta_{0}\right)+\nabla_{\vartheta} r\left(\mathbf{X}, \theta_{0}\right)\right\}\left\{K\left(\mathbf{X} ; \theta_{0}, \eta_{0}\right)-\mu_{0}\right\}\right] \\
+ & \mathbb{E}\left[(1-D)\{1-D-\pi(\mathbf{X})\}\left\{\nabla_{\vartheta} \alpha\left(\vartheta_{0}\right)+\nabla_{\vartheta} r\left(\mathbf{X}, \theta_{0}\right)\right\} \nabla_{\theta^{\top}} t\left(\mathbf{X}, \theta_{0}\right) V^{-1} A\right] \\
& \quad+\mathbb{E}\left[D \nabla_{\xi} I_{e,-1}\left\{\log f\left(Y \mid \mathbf{X}, \xi_{0}\right)\right\}{ }^{\otimes 2} I_{e}^{\top} V^{-1} A\right] \\
= & \mathbb{E}\left[\pi(\mathbf{X})\left\{\nabla_{\vartheta} \alpha\left(\vartheta_{0}\right)+\nabla_{\vartheta} r\left(\mathbf{X}, \theta_{0}\right)\right\}\left\{K\left(\mathbf{X} ; \theta_{0}, \eta_{0}\right)-\mu_{0}\right\}\right] \\
& \quad+\mathbb{E}\left[\pi(\mathbf{X})\{1-\pi(\mathbf{X})\}\left\{\nabla_{\vartheta} t\left(\mathbf{X}, \theta_{0}\right)\right\}{ }^{\otimes 2} V^{-1} A\right] \\
& \quad+\mathbb{E}\left[D \nabla_{\xi} I_{e,-1}\left\{\log f\left(Y \mid \mathbf{X}, \xi_{0}\right)\right\}{ }^{\otimes 2} I_{e}^{\top} V^{-1} A\right] \\
= & \mathbb{E}\left[\pi(\mathbf{X})\left\{\nabla_{\vartheta} \alpha\left(\vartheta_{0}\right)+\nabla_{\vartheta} r\left(\mathbf{X}, \theta_{0}\right)\right\}\left\{K\left(\mathbf{X} ; \theta_{0}, \eta_{0}\right)-\mu_{0}\right\}\right]+A_{-1},
\end{aligned}
$$

where $A_{-1}$ is $A$ without its first component and we have used the definition of $V$.

Because

$$
\begin{aligned}
& \nabla_{\vartheta} K\left(\mathbf{x} ; \theta_{0}, \lambda_{0}\right) \\
= & \frac{\int y\left\{\nabla_{\vartheta} \alpha\left(\vartheta_{0}\right)+w(\mathbf{x}, y)\right\} \lambda_{0} \exp \left(\alpha_{0}+\mathbf{x}^{\top} \beta_{0}+\gamma_{0} y\right) f\left(y \mid \mathbf{x}, \xi_{0}\right) d y}{\left(1-\lambda_{0}\right)+\lambda_{0} \exp \left\{t\left(\mathbf{x}, \theta_{0}\right)\right\}} \\
& -K\left(\mathbf{x} ; \theta_{0}, \lambda_{0}\right) \frac{\lambda_{0} \exp \left\{t\left(\mathbf{x}, \theta_{0}\right)\right\}}{\left(1-\lambda_{0}\right)+\lambda_{0} \exp \left\{t\left(\mathbf{x}, \theta_{0}\right)\right\}}\left\{\nabla_{\vartheta} \alpha\left(\vartheta_{0}\right)+\nabla_{\vartheta} r\left(\mathbf{x}, \theta_{0}\right)\right\} \\
= & \frac{1-\eta_{0}}{\eta_{0}}\{1-\pi(\mathbf{x})\} \cdot \int y\left\{\nabla_{\vartheta} \alpha\left(\vartheta_{0}\right)+w(\mathbf{x}, y)\right\} \exp \left(\alpha_{0}+\mathbf{x}^{\top} \beta_{0}+\gamma_{0} y\right) f\left(y \mid \mathbf{x}, \xi_{0}\right) d y \\
& -K\left(\mathbf{x} ; \theta_{0}, \lambda_{0}\right) \pi(\mathbf{x})\left\{\nabla_{\vartheta} \alpha\left(\vartheta_{0}\right)+\nabla_{\vartheta} r\left(\mathbf{x}, \theta_{0}\right)\right\},
\end{aligned}
$$

we have

$$
\begin{aligned}
A_{-1}= & \mathbb{E}\left\{\nabla_{\vartheta} K\left(\mathbf{x} ; \theta_{0}, \lambda_{0}\right)\right\} \\
= & \mathbb{E}\left[\frac{1-\eta_{0}}{\eta_{0}}\{1-\pi(\mathbf{x})\} \int y\left\{\nabla_{\vartheta} \alpha\left(\vartheta_{0}\right)+w(\mathbf{x}, y)\right\} \exp \left(\alpha_{0}+\mathbf{x}^{\top} \beta_{0}+\gamma_{0} y\right) f\left(y \mid \mathbf{x}, \xi_{0}\right) d y\right] \\
& \quad-\mathbb{E}\left[K\left(\mathbf{x} ; \theta_{0}, \lambda_{0}\right) \pi(\mathbf{x})\left\{\nabla_{\vartheta} \alpha\left(\vartheta_{0}\right)+\nabla_{\vartheta} r\left(\mathbf{x}, \theta_{0}\right)\right\}\right] \\
= & \left.\int\left(1-\eta_{0}\right) \int y\left\{\nabla_{\vartheta} \alpha\left(\vartheta_{0}\right)+w(\mathbf{x}, y)\right\} \exp \left(\alpha_{0}+\mathbf{x}^{\top} \beta_{0}+\gamma_{0} y\right) f\left(y \mid \mathbf{x}, \xi_{0}\right) d y\right] d F(\mathbf{x} \mid D=1) \\
- & \int K\left(\mathbf{x} ; \theta_{0}, \lambda_{0}\right)\left(1-\eta_{0}\right) \exp \left\{t\left(\mathbf{x}, \theta_{0}\right)\right\}\left\{\nabla_{\vartheta} \alpha\left(\vartheta_{0}\right)+\nabla_{\vartheta} r\left(\mathbf{x}, \theta_{0}\right)\right\} d F(\mathbf{x} \mid D=1),
\end{aligned}
$$


where we have used $t(\mathbf{x}, \theta)=\alpha+r(\mathbf{x}, \vartheta)$ and

$$
\operatorname{pr}(\mathbf{x})=\frac{\eta_{0}}{1-\pi(\mathbf{x})} \operatorname{pr}(\mathbf{x} \mid D=1) .
$$

It follows that

$$
\begin{aligned}
C_{1}= & \mathbb{E}\left[\pi(\mathbf{X})\left\{\nabla_{\vartheta} \alpha\left(\vartheta_{0}\right)+\nabla_{\vartheta} r\left(\mathbf{X}, \vartheta_{0}\right)\right\}\left\{K\left(\mathbf{X} ; \theta_{0}, \eta_{0}\right)-\mu_{0}\right\}\right]+A_{-1} \\
= & \int\left(1-\eta_{0}\right) \exp \left\{t\left(\mathbf{x}, \theta_{0}\right)\right\}\left\{\nabla_{\beta} \alpha\left(\vartheta_{0}\right)+\nabla_{\vartheta} r\left(\mathbf{x}, \vartheta_{0}\right\}\left\{K\left(\mathbf{x} ; \theta_{0}, \lambda_{0}\right)-\mu_{0}\right\} d F(\mathbf{x} \mid D=1)+\right. \\
& \left.+\int\left(1-\eta_{0}\right) \int y\left\{\nabla_{\vartheta} \alpha\left(\vartheta_{0}\right)+w(\mathbf{x}, y)\right\} \exp \left(\alpha_{0}+\mathbf{x}^{\top} \beta_{0}+\gamma_{0} y\right) f\left(y \mid \mathbf{x}, \xi_{0}\right) d y\right] d F(\mathbf{x} \mid D=1) \\
& -\int K\left(\mathbf{x} ; \theta_{0}, \lambda_{0}\right)\left(1-\eta_{0}\right) \exp \left\{t\left(\mathbf{x}, \theta_{0}\right)\right\}\left\{\nabla_{\vartheta} \alpha\left(\vartheta_{0}\right)+\nabla_{\vartheta} r\left(\mathbf{x}, \theta_{0}\right)\right\} d F(\mathbf{x} \mid D=1) \\
= & -\mu_{0} \int\left(1-\eta_{0}\right) \exp \left\{t\left(\mathbf{x}, \theta_{0}\right)\right\}\left\{\nabla_{\beta} \alpha\left(\vartheta_{0}\right)+\nabla_{\vartheta} r\left(\mathbf{x}, \vartheta_{0}\right\} d F(\mathbf{x} \mid D=1)+\right. \\
& \left.+\int\left(1-\eta_{0}\right) \int y\left\{\nabla_{\vartheta} \alpha\left(\vartheta_{0}\right)+w(\mathbf{x}, y)\right\} \exp \left(\alpha_{0}+\mathbf{x}^{\top} \beta_{0}+\gamma_{0} y\right) f\left(y \mid \mathbf{x}, \xi_{0}\right) d y\right] d F(\mathbf{x} \mid D=1) .
\end{aligned}
$$

Taking derivative with respect to $\vartheta$ on both sides of (A.18) gives

$$
0=\int\left\{\nabla_{\vartheta} \alpha\left(\vartheta_{0}\right)+\nabla_{\vartheta} r\left(\mathbf{x}, \vartheta_{0}\right)\right\} \exp \left\{\alpha\left(\vartheta_{0}\right)+r\left(\mathbf{x}, \vartheta_{0}\right)\right\} g\left(\mathbf{x}, \zeta_{0}\right) d \mathbf{x} .
$$

Since $g\left(\mathbf{x}, \zeta_{0}\right) d \mathbf{x}=\operatorname{pr}(\mathbf{x} \mid D=1) d \mathbf{x}=d F(\mathbf{x} \mid D=1)$, it follows that

$$
\left.C_{1}=\int\left(1-\eta_{0}\right) \int y\left\{\nabla_{\vartheta} \alpha\left(\vartheta_{0}\right)+w(\mathbf{x}, y)\right\} \exp \left(\alpha_{0}+\mathbf{x}^{\top} \beta_{0}+\gamma_{0} y\right) f\left(y \mid \mathbf{x}, \xi_{0}\right) d y\right] d F(\mathbf{x} \mid D=1)
$$

which is exactly $\nabla_{\vartheta} \mu\left(\theta_{0}, \eta_{0}, \zeta_{0}\right)$.

(2) Proof of Equality (A.23)

Since $\mathbb{E} \varphi_{\mu}(D, \mathbf{X}, Y)=0$, we have

$$
\begin{aligned}
C_{2}= & \mathbb{E}\left\{B_{2}(D, \mathbf{X}, Y) \varphi_{\mu}(D, \mathbf{X}, Y)\right\} \\
= & \frac{1}{\eta_{0}\left(1-\eta_{0}\right)} \mathbb{E}\left\{D \varphi_{\mu}(D, \mathbf{X}, Y)\right\} \\
= & \frac{1}{\eta_{0}\left(1-\eta_{0}\right)} \mathbb{E}\left\{D K\left(\mathbf{X} ; \theta_{0}, \eta_{0}\right)-\mu_{0} D-\pi(\mathbf{X}) D A^{\top} V^{-1} \nabla_{\theta} t\left(\mathbf{X}, \theta_{0}\right)\right. \\
& \left.+D A^{\top} V^{-1} I_{e} \nabla_{\xi} \log f\left(Y \mid \mathbf{X}, \xi_{0}\right)\right\} \\
= & \frac{1}{\eta_{0}\left(1-\eta_{0}\right)} \mathbb{E}\left\{(1-\pi(\mathbf{X})) K\left(\mathbf{X} ; \theta_{0}, \eta_{0}\right)-\mu_{0}(1-\pi(\mathbf{X}))\right. \\
& \left.-\pi(\mathbf{X})(1-\pi(\mathbf{X})) A^{\top} V^{-1} \nabla_{\theta} t\left(\mathbf{X}, \theta_{0}\right)\right\} \\
= & \frac{1}{\eta_{0}\left(1-\eta_{0}\right)}\left[\mathbb{E}\left\{(1-\pi(\mathbf{X})) K\left(\mathbf{X} ; \theta_{0}, \eta_{0}\right)\right\}-\mu_{0} \eta_{0}-A^{\top} \mathbf{e}_{1}\right],
\end{aligned}
$$


where the last equality holds because $V \mathbf{e}_{1}=\mathbb{E}\left\{\pi(\mathbf{X})(1-\pi(\mathbf{X})) \nabla_{\theta} t\left(\mathbf{X}, \theta_{0}\right)\right\}$.

Meanwhile because

$$
\begin{aligned}
A^{\top} \mathbf{e}_{1} & =\mathbb{E}\left\{\nabla_{\alpha} K\left(\mathbf{x} ; \theta_{0}, \lambda_{0}\right)\right\} \\
& =\mathbb{E}\left[\frac{1-\eta_{0}}{\eta_{0}}\{1-\pi(\mathbf{X})\} \int y \exp \left(\alpha_{0}+\mathbf{X}^{\top} \beta_{0}+\gamma_{0} y\right) f\left(y \mid \mathbf{X}, \xi_{0}\right) d y\right]-\mathbb{E}\left\{K\left(\mathbf{x} ; \theta_{0}, \lambda_{0}\right) \pi(\mathbf{x})\right\}
\end{aligned}
$$

we further have

$$
\begin{aligned}
& C_{2} \eta_{0}\left(1-\eta_{0}\right)=\mathbb{E}\left\{(1-\pi(\mathbf{X})) K\left(\mathbf{X} ; \theta_{0}, \eta_{0}\right)\right\}-\mu_{0} \eta_{0} \\
& -\mathbb{E}\left[\frac{1-\eta_{0}}{\eta_{0}}\{1-\pi(\mathbf{X})\} \int y \exp \left(\alpha_{0}+\mathbf{X}^{\top} \beta_{0}+\gamma_{0} y\right) f\left(y \mid \mathbf{X}, \xi_{0}\right) d y\right] \\
& +\mathbb{E}\left[K\left(\mathbf{X} ; \theta_{0}, \eta_{0}\right) \pi(\mathbf{X})\right] \\
& =\mathbb{E}\left\{K\left(\mathbf{X} ; \theta_{0}, \eta_{0}\right)\right\}-\mu_{0} \eta_{0} \\
& -\mathbb{E}\left[\frac{1-\eta_{0}}{\eta_{0}}\{1-\pi(\mathbf{X})\} \int y \exp \left(\alpha_{0}+\mathbf{X}^{\top} \beta_{0}+\gamma_{0} y\right) f\left(y \mid \mathbf{X}, \xi_{0}\right) d y\right] \\
& =\mu_{0}\left(1-\eta_{0}\right)-\left(1-\eta_{0}\right) \iint y \exp \left(\alpha_{0}+\mathbf{X}^{\top} \beta_{0}+\gamma_{0} y\right) f\left(y \mid \mathbf{X}, \xi_{0}\right) d y d F(\mathbf{x} \mid D=1) .
\end{aligned}
$$

Using the definition of $\mu_{0}$, we have

$$
\begin{aligned}
& C_{2}\left(1-\eta_{0}\right) \eta_{0} \\
= & \left(1-\eta_{0}\right) \int_{\mathbf{X}}\left[\int_{y} y\left\{\eta+(1-\eta) \exp \left(\alpha+\mathbf{x}^{\top} \beta+\gamma y\right)\right\} f(y \mid \mathbf{x}, \xi) d y\right] d F(\mathbf{x} \mid D=1) \\
& -\left(1-\eta_{0}\right) \iint_{y} y \exp \left(\alpha_{0}+\mathbf{X}^{\top} \beta_{0}+\gamma_{0} y\right) f\left(y \mid \mathbf{X}, \xi_{0}\right) d y \operatorname{pr}(\mathbf{x} \mid D=1) d \mathbf{x} \\
= & \left(1-\eta_{0}\right) \int_{\mathbf{X}}\left[\int_{y} y\left\{\eta_{0}-\eta_{0} \exp \left(\alpha_{0}+\mathbf{x}^{\top} \beta_{0}+\gamma_{0} y\right)\right\} f(y \mid \mathbf{x}, \xi) d y\right] d F(\mathbf{x} \mid D=1) \\
= & \left(1-\eta_{0}\right) \eta_{0} \int_{\mathbf{X}}\left[\int_{y} y\left\{1-\exp \left(\alpha_{0}+\mathbf{x}^{\top} \beta_{0}+\gamma_{0} y\right)\right\} f(y \mid \mathbf{x}, \xi) d y\right] d F(\mathbf{x} \mid D=1) .
\end{aligned}
$$

Since

$$
\nabla_{\eta} \mu=\int_{\mathbf{X}}\left[\int_{y} y\left\{1-\exp \left(\alpha+\mathbf{x}^{\top} \beta+\gamma y\right)\right\} f(y \mid \mathbf{x}, \xi) d y\right] d F(\mathbf{x} \mid D=1),
$$

we arrive at

$$
C_{2}\left(1-\eta_{0}\right) \eta_{0}=\left(1-\eta_{0}\right) \eta_{0} \nabla_{\eta} \mu\left(\theta_{0}, \eta_{0}, \zeta_{0}\right) \Longleftrightarrow C_{2}=\nabla_{\eta} \mu\left(\theta_{0}, \eta_{0}, \zeta_{0}\right) .
$$

This proves Equality (A.23). 
(3) Proof of Equality (A.24)

Since $\mathbb{E}\left\{\varphi_{\mu}(D, \mathbf{X}, Y) \mid D=1\right\}=K\left(\mathbf{X} ; \theta_{0}, \eta_{0}\right)-\mu_{0}$, we have

$$
\begin{aligned}
C_{3} & =\mathbb{E}\left\{B_{3}(D, \mathbf{X}, Y) \varphi_{\mu}(D, \mathbf{X}, Y)\right\} \\
& =\mathbb{E}\left[\nabla_{\zeta} \log g\left(\mathbf{X}, \zeta_{0}\right)\left\{K\left(\mathbf{X} ; \theta_{0}, \eta_{0}\right)-\mu_{0}\right\}\right] \\
& =\mathbb{E}\left[\nabla_{\zeta} \log g\left(\mathbf{X}, \zeta_{0}\right) K\left(\mathbf{X} ; \theta_{0}, \eta_{0}\right)\right] .
\end{aligned}
$$

Note that

$$
\mu=\mathbb{E}\left\{K\left(\mathbf{X} ; \theta_{0}, \eta_{0}\right)\right\}=\int_{\mathbf{X}} K\left(\mathbf{x}, \theta_{0}, \eta_{0}\right)\left[\eta_{0}+\left(1-\eta_{0}\right) \exp \left\{t\left(\mathbf{x}, \theta_{0}\right)\right\}\right] d F(\mathbf{x} \mid D=1),
$$

which implies

$$
\begin{aligned}
\nabla_{\zeta} \mu & =\int_{\mathbf{X}} K\left(\mathbf{x}, \theta_{0}, \lambda_{0}\right)\left[\eta_{0}+\left(1-\eta_{0}\right) \exp \left\{t\left(\mathbf{x}, \theta_{0}\right)\right\}\right]\left\{\nabla_{\zeta} \log g\left(\mathbf{x}, \zeta_{0}\right)\right\} d F(\mathbf{x} \mid D=1) \\
& =\int_{\mathbf{X}} K\left(\mathbf{x}, \theta_{0}, \lambda_{0}\right)\left\{\nabla_{\zeta} \log g\left(\mathbf{x}, \zeta_{0}\right)\right\} \operatorname{pr}(\mathbf{x}) d \mathbf{x} \\
& =\mathbb{E}\left[\nabla_{\zeta} \log g\left(\mathbf{X}, \zeta_{0}\right) K\left(\mathbf{X} ; \theta_{0}, \eta_{0}\right)\right] \\
& =C_{3} .
\end{aligned}
$$

This proves Equality (A.24) and also completes the proof of (a1).

\section{Proof of (b1)}

Consider the following function

$$
\begin{aligned}
h_{\psi}^{*}(d, \mathbf{x}, \tilde{y})= & \left\{1+\psi \varphi_{\mu}(d, \mathbf{x}, y)\right\} \times\left\{f\left(y \mid \mathbf{x}, \xi_{0}\right) \eta_{0}\right\}^{d}\left\{\exp \left(\alpha_{0}+r\left(\mathbf{x}, \vartheta_{0}\right)\right)\left(1-\eta_{0}\right)\right\}^{1-d} \\
& \times g\left(\mathbf{x}, \zeta_{0}\right) .
\end{aligned}
$$

Suppose the support of $(\mathbf{X}, Y)$ is compact, then it can be verified that the function

$$
\begin{aligned}
\varphi_{\mu}(D, \mathbf{X}, Y)= & K\left(\mathbf{X} ; \theta_{0}, \eta_{0}\right)-\mu_{0}+\{1-D-\pi(\mathbf{X})\} A^{\top} V^{-1} \nabla_{\theta} t\left(\mathbf{X}, \theta_{0}\right) \\
& +D A^{\top} V^{-1} I_{e} \nabla_{\xi} \log f\left(Y \mid \mathbf{X}, \xi_{0}\right)
\end{aligned}
$$

is bounded. Because $\mathbb{E}\left\{\varphi_{\mu}(D, \mathbf{X}, Y)\right\}=0$ where $\mathbb{E}$ takes expectation with respect to $h\left(d, \mathbf{x}, y ; \alpha_{0}, \vartheta_{0}, \eta_{0}, \zeta_{0}\right)$, the function $h_{\psi}^{*}(d, \mathbf{x}, \tilde{y})$ is a density function when $\psi$ is small enough. When $\psi=0$, it reduces to the true joint density function $h\left(d, \mathbf{x}, y ; \alpha_{0}, \vartheta_{0}, \eta_{0}, \zeta_{0}\right)$. It is easy to check that $h_{\psi}^{*}(d, \mathbf{x}, \tilde{y})$ with small enough $\psi$ is a parametric submodel and

$$
\left.\nabla_{\psi} h_{\psi}^{*}(d, \mathbf{x}, \tilde{y})\right|_{\psi=0}=\varphi_{\mu}(d, \mathbf{x}, y)
$$

This proves (b1), and hence the semiparametric efficiency of $\hat{\mu}$. 


\section{References}

Ai, C., Linton, O. \& Zhang, Z. (2018). A simple and efficient estimation method for models with nonignorable missing Data. arXiv:1801.04202v1.

Anderson, J. A. (1979). Multivariate logistic compounds. Biometrika 66, pp. 17-26.

Baker, S. G. and Laird, N. M. (1988). Regression analysis for categorical variables with outcome subject to nonignorable nonresponse. Journal of the American Statistical Association 83, pp. 62-69.

Bickel, P. J., Klaassen, C. A. J., Ritov, Y. \& Wellner, J. A. (1993). Efficient and Adaptive Estimation for Semiparametric Models. Baltimore: The Johns Hopkins University Press.

Bondell, H. D. (2007). Testing goodness-of-fit in logistic case-control studies. Biometrika 94 , pp. $487-495$.

Cai, S., Chen, J., and Zidek, J. V. (2017). Hypothesis testing in the presence of multiple samples under density ratio models. Statistica Sinica 27, pp. 761-783.

Chang, T. and Kott, P. S. (2008). Using calibration weighting to adjust for nonresponse under a plausible model. Biometrika, 95, 557-571.

Chen, J. and Liu, Y. (2013). Quantile and quantile-function estimations under density ratio model. Annals of Statistics 41, pp. 1669-1692.

Cheng, K. F. and Chu, C. K. (2004). Semiparametric density estimation under a two-sample density ratio model. Bernoulli 10, pp. 583-604.

DiCiccio, T., Hall, P., and Romano, J. (1991). Empirical likelihood is Bartlett-correctable. Annals of Statistics 19, pp. 1053-1061.

Ducharme, G. R. and Ferrigno, S. (2012). An omnibus test of goodness-of-fit for conditional distributions with applications to regression models. Journal of Statistical Planning and Inference 142, pp. 2748-2761. 
Greenlees, J. S., Reece, W. S., and Zieschang, K. D. (1982). Imputation of missing values when the probability of response depends on the variable being imputed. Journal of the American Statistical Association 7r, pp. 251-261.

Groves, R. M., Presser, S. \& Dipko, S. (2004). The role of topic interest in survey participation ecisions. Public Opinion Quarterly 68, pp. 2-31.

Hall, P. and La Scala, B. (1990). Methodology and algorithms of empirical likelihood. International Statistical Review 58, pp. 109-127.

Kenward, M. G. and Molenberghs G. (1988). Likelihood based frequentist inference when data are missing at random. Statistica Sinica 13, pp. 236-247.

Kim, J. K. and Yu, C. L. (2011). A semiparametric estimation of mean functionals with nonignorable missing data. Journal of the American Statistical Association 106, pp. 157165.

Kott, P. S. and Chang, T. (2010). Using calibration weighting to adjust for nonignorable unit nonresponse. Journal of the American Statistical Association 105, pp. 1265-1275.

Lee, B. and Marsh, L. C. (2000). Sample selection bias correction for missing response observations. Oxford Bulletin of Economics and Statistics 62, pp. 305-322.

Little, R. J. A. (1985). A note about models for selectivity bias. Econometrica 53, pp. 1469-1474.

Little, R. J. A. and Rubin, D. B. (1987). Statistical Analysis With Missing Data. New York: Wiley.

Little, R. J. A. and Rubin, D. B. (2002). Statistical Inference with Missing Data, 2nd edition. Hoboken, NJ: Wiley.

Liu, D. and Zhou, X. (2010). A model for adjusting for nonignorable verification bias in estimation of ROC curve and its area with likelihood-based approach. Biometrics 66, pp. $1119-1128$.

Louis, T. A. (1982). Finding the observed information matrix when using the EM algorithm. Journal of the Royal Statistical Society, Series B 44, pp. 226-233. 
Miao, W., Ding, P., and Geng, Z. (2016). Identifiability of normal and normal mixture models with nonignorable missing data. Journal of the American Statistical Association 111, pp. $1673-1683$.

Miao, W., Tchetgen Tchetgen, E., and Geng, Z. (2016). Identification and doubly robust estimation of data missing not at random with a shadow variable. arXiv:1509.02556.

Morikawa, K. \& Kim, J. K. (2016). Semiparametric adaptive estimation with nonignorable nonresponse data. arXiv preprint arXiv:1612.0920\%.

Morikawa, K., Kim, J. K. \& Kano, Y. (2017). Semiparametric maximum likelihood estimation with data missing not at random. The Canadian Journal of Statistics 45, pp. 393-409.

Newey, W. K. (1990). Semiparametric efficiency bounds. Journal of Applied Econometrics, 5, pp. 99-135.

Owen, A. B. (1988). Empirical likelihood ratio confidence intervals for a single functional. Biometrika 75, pp. 237-249.

Owen, A. B. (1990). Empirical likelihood ratio confidence regions. Annals of Statistics 18, pp. $90-120$.

Owen, A. B. (2001). Empirical Likelihood. New York: Chapman and Hall/CRC.

Qin, J. and Lawless, J. (1994). Empirical likelihood and general estimating equations. Annals of Statistics 22, pp. 300-325.

Qin, J., Leung, D., and Shao, J. (2002). Estimation with survey data under nonignorable nonresponse or informative sampling. Journal of the American Statistical Association 97, pp. 193-200.

Qin, J. and Zhang, B. (1997). A goodness-of-fit test for logistic regression models based on case-control data. Biometrika 84, pp. 609-618.

Riddles, M. K., Kim, J. K., and Im, J. (2016). A propensity-score-adjustment method for nonignorable nonresponse. Journal of Survey Statistics and Methodology 4, pp. 215-245. 
Robins, J. M. and Ritov, Y. (1997). Toward a curse of dimensionality appropriate (CODA) asymptotic theory for semi-parametric models. Statistics in Medicine 16, pp. 285-319.

Rubin, D. B. (1987). Multiple Imputation for Nonresponse in Surveys. New York: Wiley.

Serfling, R. J. (1980). Approximation Theorems of Mathematical Statistics. New York: Wiley.

Shao, J. (2018). Semiparametric propensity weighting for nonignorable nonresponse: a discussion of 'Statistical inference for nonignorable missing data problems: aselective review' by Niansheng Tang and Yuanyuan Ju. Statistical Theory and Related Fields 2, pp. $141-142$.

Shao, J. and Wang, L. (2016). Semiparametric inverse propensity weighting for nonignorable missing data. Biometrika 103, pp. 175-187.

Tang, G., Little, R. J. A., and Raghunathan, T. E. (2003). Analysis of multivariate missing data with nonignorable nonresponse. Biometrika 90, pp. 747-764.

Tang, N. and Ju, Y. (2018). Statistical inference for nonignorable missing-data problems: a selective review. Statistical Theory and Related Fields 2, pp. 105-133.

Tang, N., Zhao, P., and Zhu, H. (2014). Empirical likelihood for estimating equations with nonignorably missing data. Statistica Sinica 24, pp. 723-747.

Tsiatis, A. A. (2006). Semiparametric Theory and Missing Data, New York: Springer.

Wang, S., Shao, J., and Kim, J. K. (2014). Empirical distributions in selection bias models. Statistics Sinica 24, pp. 1097-116.

Zhao, J. and Shao, J. (2015). Semiparametric pseudo-likelihoods in generalized linear models with nonignorable missing data. Journal of the American Statistical Association 110, pp. $1577-1590$.

Zhao, H., Zhao, P., and Tang, N. (2013). Empirical likelihood inference for mean functionals with nonignorably missing response data. Computational Statistics and Data Analysis 66, pp. 101-116. 